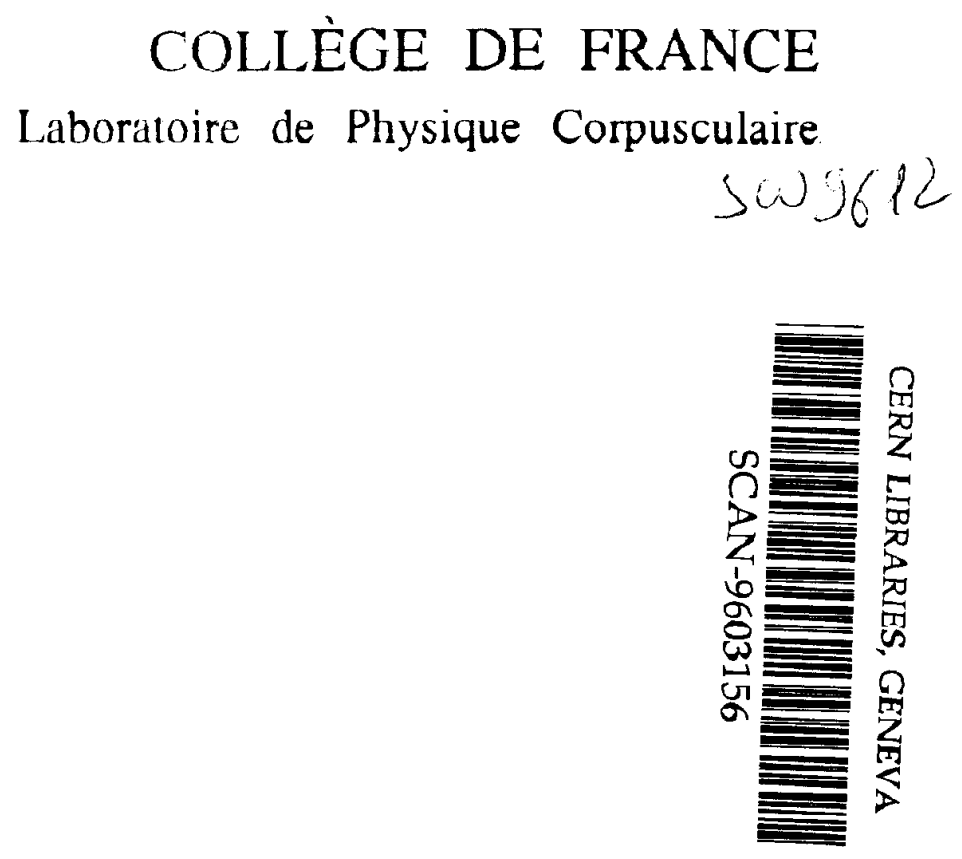

LPC 9601

A LONG BASE LINE RICH WITH A 27 kton WATER TARGET AND RADIATOR FOR DETECTION OF NEUTRINO OSCILLATIONS

T. Ypsilantis

Collège de France, Paris

A. Gougas

Johns Hopkins University

D. Hatzifotiadou

World Laboratory, Lausanne

J. Séguinot

Collège de France, Paris

COLLEGE DE FRANCE, IN2P3-CNRS, Laboratoire de Physique Corpusculaire,

11 Place Marcelin Berthelot, F-75231 Paris Cedex 05, FRANCE 


\title{
A Long Base Line RICH with a 27 kton Water Target and Radiator for Detection of Neutrino Oscillations
}

\author{
T. Ypsilantis $(a)^{*}$, A. Gougas $(b), D$. Hatzifotiadou(c) and J. Seguinot $(a)$ \\ (a) College de France \\ (b) Johns Hopkins University \\ (c) World Laboratory, Lausanne
}

\begin{abstract}
:
A 27 kton water volume is considered as a target for a long base line neutrino beam from CERN to Gran Sasso. Charged secondaries from the neutriro interactions produce Cherenkov photons in water which are imaged as rings by a sherical mirror.

The photon detector elements are 14400 photomutipliers (PMs) of $127 \mathrm{~mm}$ diameter with single photon sensitivity. A coincidence signa of $\approx 300$ PMs in time with the SPS beam burst starts readout of the PMs in bins of 1 ns cver a period of $100 \mathrm{~ns}$. This defines the effective detector granularity to be $1.44 \mathrm{Mpixeis,} \mathrm{quite} \mathrm{sufficient} \mathrm{for} \mathrm{the} \mathrm{maximum}$ expected event size of $\approx 2 \cdot 10^{4}$ photon hit points

Momentum, direction and velocity of hadrons and muons are determined from the width, center and radius of the rings, respectively. Momentum is measured if multiple scattering dominates the ring width, as is tire case for most of the particles of interest. Thresholds in water for muons, pions, kaons and protons are $0.12,0.16,0.55$ and 1.05 $\mathrm{GeV} / \mathrm{c}$, respectively.

Momentum resolutions of $: 10 \%$, mass resolutions of $5-50 \mathrm{MeV}$ and direction resolutions of $<1 \mathrm{mrad}$, are achievable.

Electrons and gammas can be measured with energy resolution $\sigma_{\mathrm{E}} / \mathrm{E}=7 \% / \sqrt{\mathrm{E}}(\mathrm{GeV})$ and with direction resolution $=1$ mrad.

The detector can be sited above ground becaust: it is directional and the SPS beam is pulsed thus it has excellent rejection of cosmic ray background.

* Corresponding author. tel. (4122) 7677577 , fax (4122) 7850207 , e-mail ypsilant @ cernvm .cern.ch
\end{abstract}




\section{Introduction}

Even though the paper delivered by T.Y at Uppsala was called "RICH Outlook" and surveyed our recent work in this area, we have decided here to concentrate on one important part of that work (Long Base Line RICH) because it is timely and even by itself fills the available space. For those interested in our CsI-Fast-RICH test beam results see [1] and for imaged aerogel, R\&D on HPDs and LHC-B see [2].

\section{1 Long Base Line RICH}

The question of neutrino masses and mixing remains one of the most important unsolved problems of particle physics. Experiments in this field use either accelerator neutrinos, solar neutrinos or atmospheric neutrinos, each sensitive to a different range of neutrino masses and mixing angles. CERN and Italy are now considering a neutrino beam traversing $731 \mathrm{~km}$ of earth to arrive at the Laboratorio Nazionale Gran Sasso (LNGS) where long base line experiments can be sited. The possibility for such experiments was already among the physics goals of the Gran Sasso Project and special care was taken to build the experimental halls aligned towards CERN [3]. The advantage of long base line neutrino experiments is, of course, their increased sensitivity to small mass differences.

For this purpose, we have proposed [4] the large water radiator and $\mathrm{RICH}$ detector shown schematically in Fig. 1. The water serves as neutrino target and Cherenkov radiating medium and is cheap and safe enough to allow large mass. The photodetectors will be visible light photomultipliers (PMs). Note that the hardware elements of this device are completely proven (clean water, phototubes, mirrors and a swimming pool of olympic volume) and require no additional R\&D.

Of course, this is the technique long used in the pioneering IMB and Kamiokande proton decay, solar neutrino and atmospheric neutrino experiments. It is also the technique which will be used in the new Super-Kamiokande $(50$ kton, 20 kton fiducial) experiment.

Our technique, however, differs in one essential aspect, namely its use of a mirror to give focused images which allow momentum, velocity and mass determination via Cherenkov images which are multiple scattering dominated (MSD). Without the mirror, image widths are dominated by track length aberrations which are of little direct physical interest but prohibit observation of multiple scattering aberrations which allow to determine momentum.

\subsection{Basic Cherenkov Relations}

The Cherenkov emission angle $\theta$ relative to the particle direction is given by the Cherenkov relation i.e $\cos \theta=1 / \mathrm{n} \beta$. The number of detected photoelectrons $N$ is given by the integral of the Frank-Tamm relation i.e 


$$
\mathrm{N}=\mathrm{N}_{0} \mathrm{Z}^{2} \mathrm{~L} \sin ^{2} \theta
$$

where $\mathrm{L}$ the particle pathlength in the medium, Ze the particle charge and $\mathrm{N}_{0}$ is the detector response parameter defined as

$$
\mathrm{N}_{0}=(\alpha / \hbar \mathrm{c}) \int(\mathrm{QTR}) \mathrm{dE}=\left(370 \mathrm{eV}^{-1} \mathrm{~cm}^{-1}\right) \mathrm{TRq}_{\text {int }}
$$

where $\alpha$ is the fine strucure constant, $E$ is the photon energy, $q_{\text {int }}=\int Q d E$ is the energy integral of $Q$ the quantum efficiency, $T$ is the radiator transmission and $R$ is the mirror reflectivity. A glass window, visible light $P M$ has $Q$ varying from 4 to $28 \%$ for $E$ from 2 to $3.5 \mathrm{eV}$. Precise integration finds $\mathrm{q}_{\mathrm{int}}=0.32 \mathrm{eV}$ thus, for $\mathrm{R}=0.95$ and $\mathrm{T}=1$ thus we expect $\mathrm{N}_{0}=112 / \mathrm{cm}$ and for $20 \%$ PM coverage $\mathrm{N}_{0}=22 / \mathrm{cm}$.

\subsection{Momentum from RICH}

It is perhaps well known that a ring image determines particle direction $\left(\theta_{p}, \phi_{p}\right)$ from the ring center and the particle velocity $\beta$ from the ring radius [5]. From the defining relation for momentum, $p=m \beta \gamma$, we obtain for the momentum error

$$
\frac{\sigma_{p}}{p}=\sqrt{\left(\frac{\sigma_{m}}{m}\right)^{2}+\left(\gamma^{2} \frac{\sigma_{\beta}}{\beta}\right)^{2}}
$$

where $m$ is assumed measured with error $\sigma_{m}$. The velocity resolution obtainable from a $\mathrm{RICH}$ is $\sigma_{\beta} / \beta \equiv \mathrm{k}_{\mathrm{r}}=\tan \theta \sigma_{\theta} / \sqrt{ } \mathrm{N}$ where $\theta$ is the measured angular radius, $\sigma_{\theta}$ is the angular width and $\mathrm{k}_{\mathrm{r}}$ is the $\mathrm{RICH}$ constant [5] thus

$$
\frac{\sigma_{p}}{p}=\sqrt{\left(\frac{\sigma_{m}}{m}\right)^{2}+\left(\gamma^{2} \frac{\tan \theta \sigma_{\theta}}{\sqrt{N}}\right)^{2}}
$$

Obviously, $m$ must be measured in order that momentum be determined morover, the above relation shows that the resolution from $\beta$ (the $2^{\text {nd }}$ term) degrades as $\gamma^{2}$ thus is rapidly limited at high momenta. Neither the momentum $\mathrm{p}$ or the momentum error $\sigma_{p} / p$ can be found without knowing the mass $m$, however to find the mass we must use the same kinematical relation $m=p / \beta \gamma$ and, by the same argument, obtain Eq. 4 in a different form i.e 


$$
\frac{\sigma_{m}}{m}=\sqrt{\left(\frac{\sigma_{p}}{p}\right)^{2}+\left(\gamma^{2} \frac{\tan \theta \sigma_{\theta}}{\sqrt{N}}\right)^{2}}
$$

Obviously, to determine $m$ we need a direct measurement of $p$ along with the RICH measurement of $\beta$.

\subsection{Multiple Scattering Dominance (MSD)}

When multiple scattering is the dominant angular error then momentum can be determined from the ring image width [6]. Since this method is new (or sufficiently old that it has been forgotten), we give here a short review of the method and its capabilities.

Historically, some early measurements of pion and muon masses were based on this effect in nuclear emulsions. In the experiment of Goldchmidt et al. [7], secondary particles produced by primary cosmic ray protons in emulsion were tracked (by human scanners looking through microscopes) and range was measured to find the particle kinetic energy via the Bethe-Bloch relation. They simultaneously measured the r.m.s angular deflection of the track to find momentum. Similar results were obtained by Camerini et al. [8] from multiple scattering and grain counting (i.e $\mathrm{dE} / \mathrm{dx} \propto 1 / \beta^{2}$ at low energies). However, most of the early measurements of pion and muon masses were from range and grain counting.

The quadratic $\gamma$ dependance of Eq.'s 4, 5 is reduced to first order if the angular width of the ring is multiple scattering dominated (MSD) i.e

$$
\sigma_{\theta}=\sigma_{\theta}(\mathrm{ms})=\frac{k_{\mathrm{ms}}}{\mathrm{p} \beta} \sqrt{\frac{\mathrm{L}^{-}}{x_{0}}}
$$

where $k_{m s}=(13.6 / \sqrt{ } 2) \mathrm{MeV}=9.6 \mathrm{MeV}$ and $x_{0}$ is the radiation length of the radiator [5]. Clearly $\sigma_{\theta}$ is proportional to $1 / \mathrm{p}$ and is formally similar to particle bending in a magnetic field (the bending angle is also $\propto 1 / p$ ). The strict similarity dissapears when we insert the $\theta$ dependance of $\beta$ and the $(\theta, N)$ dependance of $L$.

Combining Eq.'s 1, 4, 6 we obtain the momentum error of an MSD-RICH from the $\beta$ error (i.e the $2^{\text {nd }}$ term of Eq. 4 )

$$
\left(\frac{\sigma_{\mathrm{p}}}{\mathrm{p}}\right)_{\beta}=\frac{\mathrm{nK} \cos ^{2} \theta}{\mathrm{m} \sqrt{\left(\sin ^{2} \theta_{\mathrm{m}}-\sin ^{2} \theta\right)}}
$$


where $\cos \theta_{m}=1 / n$ and $K \equiv n k_{m s} / \sqrt{ }\left(N_{0} X_{0}\right)$. Thus if mass is known, Eq. 7 gives the momentum error arising from the $\beta$ measurement.

1,5 Momentum from ring radius and width when the pathlength $L$ is known

In general, momentum is determined from multiple scattering by solving Eq. 6 for $p=\left\{k_{m s} / \beta \sigma_{\theta}(m s)\right] \sqrt{ }\left(L / X_{0}\right)$. Expressed in terms of the experimental variables $\left(\theta, \sigma_{\theta}\right)$ we get

$$
\mathrm{p}=\frac{\mathrm{K}^{\prime} \cos \theta}{\sqrt{\left(\sigma_{\theta}{ }^{2}-{\sigma_{\theta 0}}^{2}\right)}}
$$

where $K^{\prime}=n k_{m s} \sqrt{ }\left(L / X_{0}\right)$ whereas $\sigma_{\theta}(m s) \equiv \sqrt{ }\left(\sigma_{\theta}^{2}-\sigma_{\theta 0^{2}}\right)$ where $\sigma_{\theta}$ is the experimental angular width and $\sigma_{\theta 0}$ is the width due to all non-momentum dependant sources. These include chromatic (E), pixel (xyz), emission point $\left(z_{e}\right)$ and impact parameter $\left(x_{e}\right)$ errors but not the energy loss or slowing error (sl) which is momentum dependant.

Here we require that $K^{\prime}$ be constant thus $L$ must be known (or measured). This is the case for most $\mathrm{RICH}$ detectors where the track pathlength $\mathrm{L}$ is determined by external trackers. But for long base line $\mathrm{RICH}$, the pathlength $\mathrm{L}$ is not (and cannot be) directly measured. This case is treated in section 1.6.

The mass defining kinematical relation $\mathrm{m}=\mathrm{p} / \beta \gamma$ may now be written in terms of the experimental variables $\left(\theta, \sigma_{\theta}\right)$ as

$$
\mathrm{m}=\mathrm{nK} \mathrm{K}^{\prime} \cos \theta \sqrt{\frac{\sin ^{2} \theta_{\mathrm{m}}-\sin ^{2} \theta}{\sigma_{\theta}^{2}-\sigma_{\theta_{0}}{ }^{2}}}
$$

Thus, from Eq.'s 8, 9 we evaluate the momentum and mass errors as

$$
\begin{aligned}
& \frac{\sigma_{p}}{p}=\sqrt{\frac{\frac{\varepsilon^{2}}{2}+\left(\tan \theta \sigma_{\theta}\right)^{2}}{N}} \\
& \frac{\sigma_{m}}{m}=\sqrt{\frac{\frac{\varepsilon^{2}}{2}+\left[\left(\gamma^{2}+1\right) \tan \theta \sigma_{\theta}\right]^{2}}{N}}
\end{aligned}
$$

where $\varepsilon \equiv \sigma_{\theta}{ }^{2} /\left(\sigma_{\theta}{ }^{2}-\sigma_{\theta 0}{ }^{2}\right) \approx 1$ for MSD but becomes large and dominant when MSD no longer applies. These derivations require an estimate [9] of the width error i.e 


$$
\sigma_{\sigma_{\theta}}=\frac{\sigma_{\theta}}{\sqrt{2 N}}
$$

1.6 Momentum from ring radius and width when $N$ is measured but $L$ is not known

Here we treat the long base line $\mathrm{RICH}$ case when the pathlength $\mathrm{L}$ is not measured but its value is obtained from Eq. 1 i.e $L=N / N_{0} \sin ^{2} \theta$. Now the momentum defining relation, Eq. 6 , written in terms of the measured variables $\left(\theta, \sigma_{\theta}, N\right)$ becomes

$$
\mathrm{p}=\left(\frac{\mathrm{K}}{\tan \theta}\right) \sqrt{\frac{N}{\sigma_{\theta}^{2}-\sigma_{\theta 0}^{2}}}
$$

where again $\mathrm{K}=n k_{\mathrm{ms}} / \sqrt{ }\left(\mathrm{N}_{0} \mathrm{X}_{0}\right)$. The mass equation $\mathrm{m}=\mathrm{p} / \beta \gamma$ now expressed in terms of the same measured variables is

$$
\mathrm{m}=\left(\frac{\mathrm{nK}}{\tan \theta}\right) \sqrt{\frac{\mathrm{N}\left(\sin ^{2} \theta_{\mathrm{m}}-\sin ^{2} \theta\right)}{\sigma_{\theta}{ }^{2}-\sigma_{\theta_{0}}{ }^{2}}}
$$

thus from Eq.'s 13, 14 we obtain for the momentum and mass errors

$$
\begin{aligned}
& \frac{\sigma_{\mathrm{p}}}{\mathrm{p}}=\sqrt{\frac{\frac{1}{4}+\frac{\varepsilon^{2}}{2}+\left(\frac{\tan \theta \sigma_{\theta}}{\sin ^{2} \theta}\right)^{2}}{N}} \\
& \frac{\sigma_{\mathrm{m}}}{\mathrm{m}}=\sqrt{\frac{\frac{1}{4}+\frac{\varepsilon^{2}}{2}+\left[\left(\gamma^{2}+\frac{1}{\sin ^{2} \theta}\right) \tan \theta \sigma_{\theta}\right]^{2}}{N}}
\end{aligned}
$$

It may be noted that the resolutions of Eq. 's 10-11 are only marginally better than Eq. 's 15-16 thus, little is lost by not having a tracker inside the water radiator volume. In fact, we have not found any reasonable way to implement such a tracker without seriously compromizing the RICH imagery. Luckily, Eq.'s 15-16 show that the impossible is also unnecessary. With $\mathrm{N} \approx 750$ points on the image (or even half that many) the $1 / \sqrt{ } \mathrm{N}$ term of Eq.'s $10-11$ and $15-16$ is small i.e $4-5 \%$ thus indicating that good resolutions are possible. 


\section{Experimental Layout}

The layout of Fig. 1 shows the proposed radiator, mirror and detector array. The mirror center of curvature $C$ determines the origin $(0,0,0)$ of the $Z X Y$ coordinate system. The water volume starting at $\mathrm{z}=6 \mathrm{~m}$ extends to $\mathrm{z}=36 \mathrm{~m}$ and transversely to $x= \pm 15 \mathrm{~m}, y= \pm 15 \mathrm{~m}$. The reflecting spherical mirror is placed at the downstream end of the cube at $\mathrm{z}=36 \mathrm{~m}$.

The PM detectors at $\mathrm{z}=16 \mathrm{~m}$ are arrayed on a flat grid $20 \mathrm{~m}$ upstream of the mirror and $10 \mathrm{~m}$ into the water target. A pixel size of $127 \mathrm{~mm} \phi$ with $20 \%$ coverage of the $30 x$ $30 \mathrm{~m}^{2}$ surface (i.e $180 \mathrm{~m}^{2}$ ) will suffice hence 14400 PMs (of $5 " \phi$ ) on a uniform $900 \mathrm{~m}^{2}$ grid. A coincidence of $\approx 300$ PM hits during the $6 \mu$ s SPS burst window (see section 5.1 ) will signal an interesting event and start readout. The PMs will be readout in bins of 1 ns for a period of $\approx 100$ ns thus increasing the effective detector granularity to 1.44 Mpixels, quite enough to image events of maximum expected size $\mathrm{N} \approx 2 \cdot 10^{4}$.

The mirror radius $\left(r_{m}=36 \mathrm{~m}\right)$ is chosen relatively small so that the image radius $\left[r_{i m} \approx f \theta \approx\left(r_{m} / 2\right)(.73) \approx 13 \mathrm{~m}\right]$ is mostly contained inside the $30 \mathrm{~m}$ wide detector array. Because the detector array is $80 \%$ transparent, the water radiator can be extended into the good optics region $10 \mathrm{~m}$ upstream of the PM plane. Photons from this region will have $16 \%$ rather than $20 \%$ imaging efficiency.

Even with only $20 \%$ detector surface coverage, an imaged hadron will have $N \approx 750$ points (see section 3.5) from an $85 \mathrm{~cm}$ long track in water (i.e one nuclear absorption length $=1 \lambda$ ). The size of $\mathrm{N}$ will be reduced by a factor .5 (or .7) if a photon absorption length of 50 (or 100) $\mathrm{m}$ is attained. Absorption losses can, of course, be compensated by additional PM coverage.

The momentum range for MSD extends up to about $4.5 \mathrm{Gev} / \mathrm{c}$, a range which includes almost all hadrons produced by $12 \mathrm{GeV}$ neutrinos via quasi-elastic and and deep inelastic charged and neutral current interactions (see section 5.4). Note that the threshold momentum for Cherenkov radiation in water is $p=1.12 \mathrm{~m}$ thus $0.12,0.16$, 0.55 and $1.05 \mathrm{GeV} / \mathrm{c}$ for muons, pions, kaons and protons, respectively. About $40 \%$ of the protons from quasi-elastic interactions are above the proton threshold (1.05 $\mathrm{GeV} / \mathrm{c}$ ). Generally, all above threshold hadrons should be well measured in long base line $\mathrm{RICH}$ i.e direction, momentum, velocity, mass and Ze (see section 4.3).

Electrons and gammas will also be well measured because an electromagnetic shower in water (radiation length $\mathrm{X}_{0}=0.36 \mathrm{~m}$ ) contains many shower electrons which radiate if $\mathrm{p}>0.57 \mathrm{MeV} / \mathrm{c}$. Thus, a Cherenkov shower is shorter than a normal ( $\mathrm{dE} / \mathrm{dx}$ sensitive) shower and is less affected by low energy fluctuations. Since it fully contained in $<5 \mathrm{~m}$ (see section 4.4) we can take as the fiducial target length $25 \mathrm{~m}$ i.e 23 ktons. The shower gives a somewhat more diffuse (but still identifiable) ring than normal with about 3000 points $/ \mathrm{GeV}$ in the image (see section 4.4). This means that water is an excellent EM calorimeter with a small stochastic term $\sigma_{E} / E \approx 7 \% / \sqrt{E}(\mathrm{GeV})$. 
In addition, the initiating shower particle direction is accurately determined ( $\leq 1$ mrad) from the center of the ring. Neutral pions and neutrons have not yet been simulated thus the capabilities of long base line $\mathrm{RICH}$ in this regard are still to be established.

Muons will also be well measured because at low momenta when the muon range is $\leq 85 \mathrm{~cm}$ (i.e 1 hadronic $\lambda$ ) then the MSD mass resolution (3-6 MeV) is good enough to distinguish muons and pions. At momenta $p>1.5 \mathrm{GeV} / \mathrm{c}$ it becomes more difficult to distinguish muons and pions by mass but then the muon range is then so long that image "lights up like a neon sign" i.e $N>5000$ is a good muon signature (compare to $\mathrm{N} \approx 750$ for pions). The momentum determination of muons in water is also good (i.e $\sigma_{\mathrm{p}} / \mathrm{p} \leq 6 \%$ for $\mathrm{p} \leq 4.5 \mathrm{GeV} / \mathrm{c}$ ). It becomes limited by emission point errors $\sigma_{\theta}\left(z_{e}\right)$ caused by the long muon pathlength in water. Extension to higher momentum $\left(\sigma_{\mathrm{p}} / \mathrm{p} \leq 10 \%\right.$ for $\left.\mathrm{p} \leq 15 \mathrm{GeV} / \mathrm{c}\right)$ can probably be obtained by application of a suitable software algorithm (see section 4.5 ).

To accurately measure muon momenta up to $40 \mathrm{GeV} / \mathrm{c}$ will require a separate $\mathrm{RICH}$ gas counter $20 \mathrm{~m}$ thick placed downstream of the water target, as shown in Fig. 1. It has a mirror radius of $40 \mathrm{~m}$ centered at $C^{\prime}$ located at $\mathrm{z}=22 \mathrm{~m}$ and the photodetector surface of radius $20 \mathrm{~m}$ measured from $C^{\prime}$. The radiator gas, $N T P C_{2} F_{6} \quad\left(\gamma_{t}=24.5\right)$, detects muons above $3 \mathrm{GeV} / \mathrm{c}$ and measures momentum to $2 \%$ at $10 \mathrm{GeV} / \mathrm{c}, 6 \%$ at $20 \mathrm{GeV} / \mathrm{c}$, $9 \%$ at $25 \mathrm{GeV} / \mathrm{c}, 13 \%$ at $30 \mathrm{GeV} / \mathrm{c}$ and $23 \%$ at $40 \mathrm{GeV} / \mathrm{c}$. Mass is also measured as shown in section 4.1. The RICH gas counter acts exactly like an analyzing magnet of a spectrometer in that it measures the momentum of the secondary particles emerging from the water target (mostly muons). The photodetectors cover $20 \%$ of the $850 \mathrm{~m}^{2}$ surface area thus $170 \mathrm{~m}^{2}$. Here, the photodetector pixel size must be $20 \times 20 \mathrm{~mm}^{2}$ thus 425 kpixels are needed. We propose to use 3300 HPDs [10] of $250 \mathrm{~mm}$ diameter each with 128 Silicon pixels of $20 \times 20 \mathrm{~mm}^{2}$. This counter is optional and would only be installed if there are strong physics arguments requiring precision measurement of high energy muons (i.e muon disappearence type experiments). Several large area trackers installed at the exit of the water radiator would measure the outgoing muon direction and an iron filter and tracker placed after the gas $\mathrm{RICH}$ would be used to further identify the muons.

\section{Properties of ring images}

Every charged particle above Cherenkov threshold will produce a ring image. Neutrals which decay into charged pairs will also form images. Electrons and gammas will shower and produce somewhat more diffuse images (see section 4.4). Gammas from $\pi^{0}$ or $\eta$ decays will also be imaged but we have not yet ascertained if these particles can be identified and reconstructed. High energy neutrons will $n-p$ scatter and 
produce proton recoils which are imaged if they are above threshold. A large effort of simulation and analysis will be required to determine the limits of RICH imagery.

The optics of spherical mirrors combined with the fact that Cherenkov light rays in any plane containing the track form parallel bundles and thus can be focused to a point. Contributions from all these planes form a ring on the mirror focal surface. In addition, it is easy to show that parallel particles form the same ring image thus the ring center determines the particle direction $\left(\theta_{p}, \varphi_{p}\right)$ [5].

An important advantage of $\mathrm{RICH}$ is that the Cherenkov angle distribution is Gaussian without the Landau tail which characterizes $\mathrm{dE} / \mathrm{dx}$ devices.

\subsection{Parameters of the image}

The parameters of the ring image are the photon detection point $(x, y, z)$, the photon emission point $\left(z_{e}, x_{e}\right)$, the particle direction $\left(\theta_{p}, \varphi_{p}\right)$ and the photon energy $E$. The photon emission point $z_{\mathrm{e}}$ is measured along the particle track and the impact parameter $x_{e}$ is the perpendicular distance to the track from the mirror center of curvature $C$ as shown in Fig. 2. Note that two coordinate systems are in use: The ZXXY system is the fixed space coordinate system as in Fig.1; the second PQR coordinate sytem is defined from $C$ such that $P$ is parallel to the track and $Q$ intersects the track thus $\left(z_{e}, x_{e}, 0\right)$ is the photon emission point in the PQR system.

Six of the eight variables $\left(z, x, y, x_{e}, \theta_{p}, \varphi_{p}\right)$ can be arbitrarily well measured with the group $\left(z, x, y, \theta_{p}, \varphi_{p}\right)$ depending only on the precision of the photon detector while $x_{e}$ depends on the tracker. The photon emission distance $z_{e}$ has an irreducible error

$$
\sigma_{z_{e}}=\frac{\Delta z_{e}}{\sqrt{12}}
$$

which can only be improved by reducing the radiating pathlength $\mathrm{L}=\Delta \mathrm{z}_{\mathrm{e}}$. The error in $\theta$ due to errors in any of the variables $v_{i}=\left(z_{e}, x_{e}, z, x, y, \theta_{p}, \varphi_{p}\right)$ may be expressed as

$$
\sigma_{\theta}\left(v_{i}\right)=\left(\frac{\partial \theta}{\partial v_{i}}\right) \sigma_{v_{i}}
$$

where $\partial \theta / \partial v_{i}$ is calculated from the reconstruction relation $\theta=\theta\left(v_{i}\right)$. The energy error for a square detector response is

$$
\sigma_{\mathrm{E}}=\frac{\Delta \mathrm{E}}{\sqrt{12}}
$$


and can only be improved by reducing the energy bandwidth $\Delta \mathrm{E}$. The corresponding Cherenkov angle error is

$$
\sigma_{\theta}(E)=\left(\frac{\partial \theta}{\partial n}\right)\left(\frac{d n}{d E}\right) \sigma_{E}
$$

where $n(E)$ gives the radiator dispersion. The errors $\left(\sigma_{z_{\mathrm{e}}}, \sigma_{\mathrm{E}}\right)$ determine the RICH resolution limits. An analytic evaluation of $\sigma_{\theta}\left(v_{i}\right)$ via the reconstruction relation $\theta=\theta\left(v_{i}\right)$ may be found in [5].

\subsection{Impact parameter $x_{e}$, interaction point $z_{e v}$ and emission point $z_{e}$ determination}

If tracker data were available and a minimum of two tracks were observed then an interaction vertex $\left(x_{v}, y_{v}, z_{v}\right)$ can be found. Assume that at least two tracks come from a vertex point $\left(z_{v}, x_{v}, y_{v}\right)$ and that this point is measured as are the track directions $\left(\theta_{p}\right.$, $\varphi_{p}$ ). The impact parameter, along the $Q$ axis in the plane $\varphi=\varphi_{p}$ (see Fig. 2 ), is then determined as

$$
x_{e}=z_{v} \sin \theta_{p}-\rho_{v} \cos \theta_{p}
$$

where $\rho_{v}=\sqrt{ }\left(x_{v}{ }^{2}+y_{v}{ }^{2}\right)$. Similariy the vertex distance along the track is

$$
z_{e v}=z_{v} \cos \theta_{p}+\rho_{v} \sin \theta_{p}
$$

where $z_{e}=0$ is the point where the $Q$ axis intersects the track (normally).

Obviously, the photon emission point $z_{e}$ cannot be measured but the precise emission point is much less critical (focusing is defined by the condition $\partial \theta / \partial z_{\mathrm{e}}=0$ ). The best we can do is assume it to be equidistant between $\mathrm{z}_{\mathrm{ev}}$ and $\mathrm{z}_{\mathrm{et}}$.

$$
z_{e}=\frac{z_{e v}+z_{e t}}{2}
$$

where for muons which reach the mirror $z_{e t}=\sqrt{ }\left(r_{m}{ }^{2}-x_{e}{ }^{2}\right)$ and for a hadron $z_{e t} \approx z_{e v}+\lambda$.

Long base line RICH, however, will not have a tracker but $\left(\theta_{p}, \varphi_{p}\right)$ will be known from the ring image center hence the plane of the impact parameter is known i.e $\varphi=\varphi_{p}$. The magnitude of the impact parameter $x_{e}$ can be found by measuring time. We find from geometry and optics that the arrival time $t_{i}$ of the ith photon of the ring is 


$$
\begin{aligned}
& \frac{\mathrm{ct}_{\mathrm{i}}}{\mathrm{n}}=-\mathrm{z}_{\mathrm{ev}} \cos \theta-\mathrm{x}_{\mathrm{e}} \sin \theta \cos \varphi_{\mathrm{i}} \\
& +2 \sqrt{\mathrm{r}_{\mathrm{m}}{ }^{2}-\mathrm{r}_{\mathrm{e}}{ }^{2}+\left(\mathrm{z}_{\mathrm{e}} \cos \theta+\mathrm{x}_{\mathrm{e}} \sin \theta \cos \varphi_{\mathrm{i}}\right)^{2}} \\
& -\sqrt{\mathrm{r}^{2}-\mathrm{r}^{2}+\left(\mathrm{z}_{\mathrm{e}} \cos \theta+\mathrm{x}_{\mathrm{e}} \sin \theta \cos \varphi_{\mathrm{i}}\right)^{2}}
\end{aligned}
$$

where $t_{i}$ is measured from the time of track creation at point $z_{e v}$. Since this absolute time is not known we can only measure the time difference between $t_{i}$ and some reference photon $t_{0}$ of the ring (say at $\varphi=0$ ). We have verified, numerically, that to $10 \%$ accuracy

$$
x_{\mathbf{e}_{i}}=\frac{c\left(t_{i}-t_{0}\right)}{n \sin \theta\left(1-\cos \varphi_{i}\right)}
$$

i.e each photon point gives a measure of $x_{e}$. The full image therefore gives the average

$$
x_{e}=\frac{1}{N} \sum_{i=1}^{N} x_{e_{i}}
$$

from $\mathrm{N}$ time measurements.

If two images are observed and their impact parameters are found then a single constraint relates these impact parameters and, when satisfied, guarantees that the tracks come from a common vertex. In fact if $M$ images are seen, the number of contraints relating their impact parameters is $C=2 \mathrm{M}-3$.

The unit vector $\vec{a}$ along the track and parallel to the $P$ axis has components $\left(a_{z}, a_{x}\right.$, $\left.a_{y}\right)$ where $a_{z}=\cos \theta_{p}, a_{x}=\sin \theta_{p} \cos \varphi_{p}, a_{y}=\sin \theta_{p} \sin \varphi_{p}$. The unit vector $\vec{b}=\left(b_{z}, b_{x}, b_{y}\right)$ along the impact parameter axis $Q$ (of Fig. 2) has components $b_{z}=-a_{x}, b_{x}=\sin ^{2} \varphi_{p}+\cos \theta_{p} \cos ^{2} \varphi_{p}$, $b_{y}=\left(\cos \theta_{p}-1\right) \cos \varphi_{p} \sin \varphi_{p}$. The unit vector normal to $\vec{a}$ and $\vec{b}$ is $\vec{c}=\vec{a} \times \vec{b}$ has $c_{z}=-a_{y}$, $c_{x}=b_{y}, c_{y}=\cos ^{2} \varphi_{p}+\cos \theta_{p} \sin ^{2} \varphi_{p}$. For two images (labeled 1 and 2 ) we can show that the distances along $\overrightarrow{\mathbf{a}}$ to the vertex point (in PQR system) are

$$
\begin{aligned}
& z_{e_{1}}=\alpha_{22} x_{e_{2}}-\alpha_{21} x_{e_{1}} \\
& z_{e_{2}}=\alpha_{12} x_{e_{2}}-\alpha_{11} x_{e_{1}}
\end{aligned}
$$

where $x_{e 1}$ and $x_{e 2}$ are impact parameters found from timing (i.e Eq.'s 25, 26) and $\alpha_{22}=\left(a_{2 z} b_{2 x}-a_{2 x} b_{2 z}\right) / D, \alpha_{21}=\left(a_{2 z} b_{1 x}-a_{2 x} b_{1 z}\right) / D, \alpha_{12}=\left(a_{1 z} b_{2 x}-a_{1 x} b_{2 z}\right) / D, \alpha_{11}=\left(a_{1 z} b_{1 x}-a_{1 x} b_{1 z}\right) / D$ with $\mathrm{D}=\mathrm{a}_{2 z} \mathrm{a}_{1 \mathrm{x}}-\mathrm{a}_{2 \mathrm{x}} \mathrm{a}_{12}$. The constraint relation 


$$
\frac{x_{e_{2}}}{x_{e_{1}}}=\frac{\alpha_{11} a_{2 y}-\alpha_{21} a_{1 y}+b_{1 y}}{\alpha_{12} a_{2 y}-\alpha_{22} a_{1 y}+b_{2 y}}
$$

will be satisfied if the tracks come a single vertex (or decay or interaction) point. If so, that point $\left(z_{v}, x_{v}, y_{v}\right)$ is given by the vector equation

$$
\vec{r}_{v}=x_{e_{2}} \vec{b}_{2}+z_{e v_{2}} \vec{a}_{2}=x_{e_{1}} \vec{b}_{1}+z_{e v_{1}} \vec{a}_{1}
$$

Note that for $M=3$ images there are $C=3$ constraints thus a $\chi^{2}$ minimization will strongly improve the vertex determination (even the $C=1$ constraint will help). We estimate that the vertex point will be determined with $\mathrm{cm}$ like accuracy although this has not yet been demonstrated by simulation.

The choice of $z_{\mathrm{e}}$ is obtained as before from Eq. 23 et. seq.

\subsection{Reconstruction of the Cherenkov angle}

A general method to reconstruct the Cherenkov angle $\theta$ from the hit point $(x, y, z)$ requires that the particle impact parameter $x_{e}$ and the emission point $z_{e}$ along the track be known [5]. Using the values of $\left(x_{e}, z_{e}\right)$ found (see section 3.2) with the detected photon coordinates $(x, y, z)$, with $r=\sqrt{ }\left(x^{2}+y^{2}+z^{2}\right)$, we obtain the generalized detected angle $\Omega$ (see Fig. 2) from the relation

$$
\cos \Omega=\frac{\left(z z_{e}+x x_{e}\right) z_{p}+\left(x z_{e}-z x_{e}\right) x_{p}}{r_{e}}
$$

where $r_{e}=\sqrt{ }\left(x_{e}{ }^{2}+z_{e}{ }^{2}\right)$ (by construction $\left.y_{e}=0\right), z_{p}=\cos \theta_{p}$ and $x_{p}=\sin \theta_{p}$. We then find the generalized emission angle $\Omega_{e}$ (see Fig. 2 ) by numerical inversion of the equation

$$
\Omega=\Omega_{e}+\arcsin \left(\frac{r_{e} \sin \Omega_{e}}{r}\right)-2 \arcsin \left(\frac{r_{e} \sin \Omega_{e}}{r_{m}}\right)
$$

with $\Omega$ from Eq. 30. Knowing $\Omega$ and $\Omega \mathrm{e}$ we can then reconstruct the original Cherenkov emission angles $(\theta, \phi)$ from the relations 


$$
\begin{aligned}
& \cos \theta=\frac{\left(z z_{p}+x x_{p}\right) \sin \Omega_{e}}{r \sin \Omega}-\frac{z_{e} \sin \Omega^{\prime}}{r_{e} \sin \Omega} \\
& \sin \theta \cos \varphi=\frac{\left(x z_{p}-z x_{p}\right) \sin \Omega_{e}}{r \sin \Omega}-\frac{x_{e} \sin \Omega^{\prime}}{r_{e} \sin \Omega} \\
& \sin \theta \sin \varphi=\frac{y \sin \Omega_{e}}{r \sin \Omega}
\end{aligned}
$$

where $\Omega^{\prime}=\Omega_{\mathrm{e}}-\Omega$.

In Fig. 3 we show an event generated by a $40 \mathrm{GeV} / \mathrm{c} v_{\mu}$ in an Argon gas radiator $\left(\gamma_{\mathrm{t}}=10\right)$ with $25 \mathrm{~m}$ pathlength giving $\approx 2000$ points on the images (taken from an earlier detector setup [6] and used here to illustrate a point). This interaction produced only three above threshold particles as labeled in Fig. 3. Pattern recognition is easy, even visually. The generated $\theta$ distributions are shown in Fig. 4 along with the reconstructed $\theta$ distributions (from Eq.'s 30-32) for tracks 2 and 3 of Fig.3. Note that the reconstructed distributions are as sharp as the generated distributions (or even sharper due to pattern recognition cuts). Note also that track 2 has a very wide $\theta$ distribution (MSD) whereas track 3 is intrinsic (beyond MSD).

\subsection{Number of points on the image}

As shown in section 1.2 and Eq. 5, the response factor $N_{0}=(R)(f)\left(q_{\text {int }}\right)\left(370 \mathrm{eV}^{-1} \mathrm{~cm}^{-1}\right)$. For $\mathrm{f}=20 \%$ surface cover and an $\mathrm{R}=95 \%$ mirror and visible light $P M$ s with $\mathrm{q}_{\text {int }}=.318 \mathrm{eV}$ thus $N_{0}=22 \mathrm{~cm}^{-1}$. For a $\beta=1$ particle in water with $L=85 \mathrm{~cm}$ (i.e $1 \lambda$ ) we expect $N \approx 830$ photoelectrons points per image. This will be reduced to $\approx 400$ for the average photon pathlength of $35 \mathrm{~m}$ and a water absorption length of $50 \mathrm{~m}$.

\section{Long base line RICH performance}

\subsection{Particle momentum resolution}

We evaluate resolutions $\sigma_{\mathrm{p}} / \mathrm{p}$ and $\sigma_{\mathrm{m}}$ for tracks with $\theta_{\mathrm{p}}=0$ in the detector layout of Fig. 1. The pixel sizes are $\Delta x=\Delta y=125 \mathrm{~mm}, \Delta z=1 \mathrm{~mm}, \Delta \mathrm{t}=2$ ns corresponding to $\Delta \mathrm{x}_{\mathrm{e}}=20$ $\mathrm{mm}$. The refractive index and dispersion function $\mathrm{n}(\mathrm{E})$ of water are taken from [11].

In Fig. 5 we show the various contributions to the angular error $\sigma_{\theta}$ vs $x_{e}$ for a 1 $\mathrm{GeV} / \mathrm{c}$ pion with $85 \mathrm{~cm}$ pathlength $(1 \lambda)$. We note that the dominant contrIbutor is multiple scattering $\sigma_{\theta}(\mathrm{ms})=15 \mathrm{mrad}$ (i.e MSD) while chromatic $\sigma_{\theta}(E)=3.6 \mathrm{mrad}$, pixel $\sigma_{\theta}(x y z)=1.9 \mathrm{mrad}$ and energy loss $\sigma_{\theta}(\mathrm{sl})=0.4 \mathrm{mrad}$ (i.e. slowing) are less important. As seen in Fig. 5, only $\sigma_{\theta}\left(x_{e}\right)$ and $\sigma_{\theta}\left(z_{e}\right)$ vary with $x_{e}$ but are not yet significant even for $x_{e}$ as large as $15 \mathrm{~m}$. 
Fig. 6 shows the momentum resolution $\sigma_{\mathrm{p}} / \mathrm{p}$ from Eq. 15 and photon number $\mathrm{N}$ vs $x_{e}$ for a $1 \mathrm{GeV} / c$ pion. The loss in momentum resolution with $x_{e}$ is due to the loss of imaged points $\mathrm{N}$, in accord with Fig. 5 which shows that the $\mathrm{x}_{\mathrm{e}}$ errors are small.

In Fig. 7 we give the resolution $\sigma_{p} / p$ vs $p$ for $\mu, \pi, K, P$ for muon pathlengths of 15 $\mathrm{m}$ and for hadron pathlengths of $0.85 \mathrm{~m}$ (i.e $1 \lambda$ ). The solid curves are from Eq. 15 (for MSD) whereas the dot-dash curves are from Eq. 7 (i.e from the $\beta$ measurement when mass is known). Note that the solid curve resolutions are excellent i.e between 1 and $6 \%$ for $\mathrm{p} \leq 5 \mathrm{GeV} / \mathrm{c}$. For kaons and protons the dot-dash curves are everywhere $<1 \%$ and better than the solid curves whereas for pions and muons they are better only below 1.3 and $0.5 \mathrm{GeV} / \mathrm{c}$, respectively.

Fig. 8 shows the mass resolution from combined $\mathrm{ms}$ and $\beta$ measurements (Eq. 16). For $\mathrm{p} \leq 1.25 \mathrm{GeV} / \mathrm{c}$ the resolution $\sigma_{\mathrm{m}} \approx 5-7 \mathrm{MeV}$ is sufficient for $\mu / \pi \mathrm{ID}$. Above $1 \mathrm{GeV} / \mathrm{c}$ the muon range becomes so long that the muon is identified by the large value of $N>$ 5000. The $\mathrm{K}$ resolution, $\sigma_{\mathrm{m}} \approx 20-30 \mathrm{MeV}$, is sufficient for $\pi / \mathrm{K}$ ID and the proton resolution, $\sigma_{m} \approx 50-60 \mathrm{MeV}$, suffices for $\mathrm{K} / \mathrm{P}$ ID. Therefore, the combined $\mathrm{ms}$ and $\beta$ measurements allow to identify all stable particles and so we are free to choose the best resolution curves of Fig. 7 (solid or dot-dash).

Fig. 9 shows the performance of the $20 \mathrm{~m}$ thick RICH gas counter (NTP $\mathrm{C}_{2} \mathrm{~F}_{6}$, $\gamma_{\mathrm{t}}=24.5$ ) for muons of 3 to $40 \mathrm{GeV} / \mathrm{c}$ via $\mathrm{Eq}$. 7. The mass resolution shows that $\sigma_{\mathrm{m}} \approx 10$ $\mathrm{MeV}$ (Eq. 16), this is marginal for $\mu / \pi$ ID however very few hadrons or electrons get out of the water. An additional iron filter placed after the RICH gas counter would guarantee adequate muon ID. The momentum resolution $\sigma_{\mathrm{p}} / \mathrm{p}$ is $2 \%$ at $10 \mathrm{GeV} / \mathrm{c}, 9 \%$ at $25 \mathrm{GeV} / \mathrm{c}$ and $23 \%$ at $40 \mathrm{GeV} / \mathrm{c}$ thus, gas $\mathrm{RICH}$ is equivalent to a powerful magnet.

\subsection{Particle direction determination}

The polar angles $\left(\theta_{p}, \phi_{p}\right)$ of a particle producing a ring image are determined with high precision from the ring center i.e $\sigma_{\theta p} \approx \sigma_{\phi p} \approx \sigma_{\theta} / \sqrt{N}$. For a $1 \mathrm{GeV} / \mathrm{c}$ pion track in water with Cherenkov pathlength of $85 \mathrm{~cm}$ we have $\sigma_{\theta} \approx 15 \mathrm{mrad}$ and $N \approx 400$ hence $\sigma_{\theta p} \approx \sigma_{\phi p} \approx 0.75 \mathrm{mrad}$. The direction error for electrons, gammas and muons should be at least as good because $\mathrm{N}$ is considerably greater.

\subsection{Hadron images}

In Fig.'s 10 we show images of $1 \mathrm{GeV} / \mathrm{c}$ pions for an on-axis vertex at $z_{\mathrm{v}}=20.75$. The conditions of the simulation were $\theta_{p}=0$ and $x_{e}=0$ in the geometry of Fig. 1. All resolution errors $\sigma_{\theta}$ were set to zero except $\sigma_{\theta}\left(z_{e}\right)$ and these were generated by choosing values of $z_{e}$ uniformly distributed between $z_{e v}$ and $z_{e v}+\lambda$. The transverse radiator size was taken to be $x=y= \pm 20 \mathrm{~m}$ to allow displaced images to be seen in full. The binning of the imaged points $\Delta x=\Delta y=0.4 \mathrm{~m}$ is, in fact, bigger than the long base line RICH pixel 
size $\Delta x=\Delta y=0.125 \mathrm{~m}$ and approximates the expected $\mathrm{ms}$ error. Similar images were obtained at $\mathrm{z}_{\mathrm{ev}}=10.75$ and $15.75 \mathrm{~m}$ but are not shown. As can be seen the image is sharp and we found that radius of the image changed little for a $\mathrm{z}_{\mathrm{ev}}$ variation of $10 \mathrm{~m}$. The imaged radii are $12.63,12.49$ and $12.66 \mathrm{~m}$ for $\mathrm{z}_{\mathrm{ev}}=10.75,15.75$ and $20.75 \mathrm{~m}$, repectively.

In Fig. 11 we see the simulated image of an on-axis $1 \mathrm{GeV} / \mathrm{c}$ pion created at $z_{v}=20.75 \mathrm{~m}$ with $\theta_{\mathrm{p}}=300 \mathrm{mrad}$ thus $x_{\mathrm{e}}=6.1 \mathrm{~m}$ (see Eq. 21). The image would have been cutoff at $y=15 \mathrm{~m}$ in the geometry of Fig. 1 but only $\approx 20 \%$ of the image would have been lost. We note that the image is still sharp and suitable for pattern recognition, This is the essential criterion because if $x_{e}$ and $z_{e}$ are measured (section 3.2) then the reconstruction procedure (section 3.3) removes all "out of focusness" and leaves only essential abberations (i.e Fig. 5) and restores MSD.

The position of a $\mathrm{RICH}$ image in the detector is determined by the particle direction $\theta_{\mathrm{p}}$ thus a displacement $\Delta x= \pm 15 \mathrm{~m}$ would still leave half the ring within detector grid. Therefore, the angular range of acceptance is $\tan \left(\Delta \theta_{\mathrm{p}}\right) \approx x / z_{\mathrm{d}}$ which for $x= \pm 15 \mathrm{~m}$ and for the detector grid at $z_{d}=16 \mathrm{~m}$ implies that our acceptance $\Delta \theta_{p}$ is $\approx 750$ mrad.

This acceptance could be further increased if the walls of the cube (say at $\pm w$ ) are made reflective. This, however, introduces a pattern recognition problem since the part of the ring which is reflected would have focused outside $w$ at point $x=w+d$ and detected at $x_{d}=w-d=x-2 d$ (similarly for $y$ ). This pattern problem may be resolvable but for the moment and pending further study, we assume no reflections. Of course, the practical problem of making all the walls reflective may be the decisive element.

\subsection{Electron and gamma images}

We made a full GEANT simulation of electron showers in water for 1,3 , and 5 $\mathrm{GeV} / \mathrm{c}$ electrons starting at $z_{\mathrm{v}}=25.2 \mathrm{~m}$ with $\theta_{\mathrm{p}}=0$ and $\mathrm{x}_{\mathrm{e}}=0$ in the geometry of Fig. 1 . We took the GEANT output for the shower electrons as the input to our program which simulates ring images with, of course, ms capability.

Fig. 12 shows images of 10 electrons of $5 \mathrm{GeV}$ (we reduced $\mathrm{N}$ by 10 for each event so 10 events gives the right value i.e $\mathrm{N}=16800 \pm 500$ but smooths the distributions). The image is quite easily recognizable.

In Fig. 13 we see the radius distribution with a FWHM $=200 \mathrm{~cm}$ thus $\sigma_{\theta} \approx 48 \mathrm{mrad}$ i.e much larger than for hadron images. Moreover the tails at small radii are significant and will be valuable for further identification of electrons or gammas.

Fig.14 shows a single event (with $\mathrm{N}$ reduced by 10 ). Note that its width and tails agree with the smoothed distribution of Fig. 13.

Fig. 15 is a visual image of the showering tracks in depth.

In Fig. 16 we show the number of hits from showering electrons vs depth $Z$. It is surprising to note that almost all of the shower is largely spent in $5 \mathrm{~m}$ (i. e $13.9 \mathrm{X}_{0}$ ) and 
completely spent in $6.2 \mathrm{~m}$ (i. e $17.2 \mathrm{X}_{0}$ ). Thus we will require that neutrino interactions occur in the first $25 \mathrm{~m}$ (fiducial) leaving the last $5 \mathrm{~m}$ to contain an EM shower.

The simulation gave values of $\mathrm{N}=3150 \pm 300,10200 \pm 300$ and $16500 \pm 500$ for electron energies $E=1,3$ and $5 \mathrm{Gev}$, respectively. The ratios $N / E$ are $3150 \pm 300,3400 \pm 100$ and $3360 \pm 100$, respectively hence the average $N / E=3350$. Values for the stochastic term $\left(\sigma_{E} / E\right) \sqrt{ } \mathrm{E}$ are $9.5 \%, 5.2 \%$ and $6.8 \%$, respectively thus the average EM energy resolution in water is

$$
\frac{\sigma_{\mathrm{E}}}{\mathrm{E}}=\frac{7.2 \%}{\sqrt{\mathrm{E}(\mathrm{GeV})}}
$$

\subsection{Muon images}

Muon images have not been simulated but from analytical calculations we know that at $2.25 \mathrm{GeV} / \mathrm{c}$ the image widths $\sigma_{\theta}(\mathrm{ms}) \approx \sigma_{\theta}\left(\mathrm{z}_{\mathrm{e}}\right)=26 \mathrm{mrad}$. Beyond this point and up to $4.5 \mathrm{GeV} / \mathrm{c}$ the $\mathrm{ms}$ contribution is still large enough that $\sigma_{\theta}\left(z_{\mathrm{e}}\right)$ can be subtracted in quadrature leaving a still significant $\mathrm{ms}$ value (see Fig. 7 ).

Above $4.5 \mathrm{GeV} / \mathrm{c}$ the ms contribution becomes so small that $\sigma_{\theta}\left(z_{\mathrm{e}}\right)$ dominates $(\approx 36$ mrad for $15 \mathrm{~m}$ pathlength) and we must adopt a different strategy. We know that any track with $N>5000$ (without the width and tails of an EM shower) must be a muon thus, its mass is determined. If the track is accompanied by a second track and both impact parameters are found by timing (Eq.'s 25-26) then their common vertex point can also be found (Eq.'s 27-29). We then can find the muon pathlength to the mirror ( $N$ also gives a good estimate of this length). Taking ze at the track center (Eq. 23) and $x_{e}$ as described above, we reconstruct the Cerenkov angle distribution from Eq.'s 30-32. Since $\sigma_{\theta}\left(z_{e}\right)$ is dominant, the reconstructed $\theta$ distribution will order the photon emission points along the track. This process effectively splits up a long track into many shorter ( $\approx 0.85 \mathrm{~m}$ long) segments which are individually not dominated by $\sigma_{\theta}\left(z_{e}\right)$. When the photons of a segment are reconstructed using its central $z_{e}$ value, each $\theta$ distribution narrows and we obtain a momentum resolution dominated by intrinsic errors. Statistically this is equivalent to $15 / .85 \approx 18$ separate measurements of momentum each of $\approx 50 \%$ accuracy for a an overall expected value $\approx 12 \%$ at $15 \mathrm{Gev} / \mathrm{c}$.

\section{Beams and sites}

\subsection{The SPS extracted beam}

The SPS beam operates at $200 \mathrm{MHz}$ thus with a $5 \mathrm{~ns}$ periodicity. This means that succeeding $\mathrm{rf}$ bunches are separated in the water target by only $1.5 \mathrm{~m}$ hence absolute timing cannot determine the interaction vertex point (this would be possible if the rf bunches were separated by $100 \mathrm{~ns}$ ). In other words, at any given time within an SPS burst there will be $20 \mathrm{rf}$ bunches inside the water target. 
One, two or three SPS beam bursts can be extracted every SPS cycle of $10 \mathrm{sec}$. If one burst is extracted it is $23 \mu \mathrm{s}$ long and contain $1 \cdot 3 \cdot 10^{13} \mathrm{p}$. If two bursts are extracted they are $10 \mu \mathrm{s}$ long separated by $50 \mathrm{~ms}$ and each with $1.3 \cdot 10^{13} \mathrm{p}$ for a total of $2.6 \cdot 10^{13} \mathrm{p} / \mathrm{SPS}$ cycle. When three bursts are extracted they are $6 \mu \mathrm{s}$ long separated by $50 \mathrm{~ms}$ with a total of $3.9 \cdot 10^{13}$ p. Assuming a $30 \%$ SPS duty factor we expect $10^{6}$ SPS cycles/y thus from 1.3 to $3.9 \cdot 10^{19}$ protons on target (p.o.t.)/y [12].

\subsection{The Neutrino beam and event rates}

The neutrino beam is designed to give a flux of 500 events $/$ kton-y for $10^{19}$ p.o.t./y [13]. Since we can expect up to $3.9 \cdot 10^{19}$ p.o.t./y (see above), the flux rises to $\approx 2000$ events/kton-y. Since long base line RICH will contain 23 fiducial ktons of water, we will amass up to 45000 events/y (for no oscillations i.e for $\Delta \mathrm{m}^{2}=0, \sin ^{2} 2 \theta=1$ ).

\subsection{Where to site long base line RICH}

As is clear from Fig. 1, the Gran Sasso tunnel would have to be $43 \mathrm{~m}$ in diameter to contain a $30 \mathrm{~m}$ cube. This is twice the diameter of the present and future Gran Sasso tunnels so we have investigated the operation of long base line RICH above ground.

As a worse case scenario we take the full cosmic ray flux (including the soft component) given by the PDG [14] of $180 / \mathrm{m}^{2}$-s. For the long base line RICH surface area $\left(900 \mathrm{~m}^{2}\right)$ we obtain a rate $0.16 \mathrm{MHz}$. Thus, during a beam burst of $6 \mu$ s we expect $\approx 1$ muon to traverse long base line RICH. Since each $6 \mu \mathrm{s}$ burst delivers $\approx 10^{13}$ p.o.t., the probability for a real event is $\left(500 / 10^{6}\right) \times 27=.0135$ i.e one real event $(S)$ per 74 openings. During this opening we expect $\approx 1$ through-going muon $(B)$ thus $S / B \approx 1$. The other 73 openings produce only obvious background which cannot be confused with the signal since it lies in another burst.

Several methods are available to reduce this background to a negligable level. Firstly by optically shielding the PMs so that they view only the mirror. Secondly by timing, since to determine $x_{e}$, the PM hits will be binned in buckets of 1 or 2 ns width. This will readily distinguish background from signal photons which arrive with $\leq 100$ ns dispersion (in $6 \mu \mathrm{s}$ ) thus a potential background reduction factor of $\approx 60$. Thirdly from the track patterns, since the background is mostly vertical (and does not form images) whereas the signal is mostly longitudinal (and does form images). Lastly by shielding, even though long base line $\mathrm{RICH}$ will be above ground it should be placed behind the mountain (when viewed from CERN) thus screening out the more horizontal muon tracks. All of these reduction factors may not be necessary and further study will be required to choose the right mix. 


\subsection{Event Distributions}

In Fig.'s 17-20 we show the $p$ and $\theta_{p}$ distributions for pions and protons (from the generator Pythia 5.7) for $12 \mathrm{GeV} / \mathrm{c} v_{\mu} \mathrm{s}$ interacting via charged and neutral currents. The solid curves show all particles and the dotted curves show those above threshold.

The momentum distribution of pions is shown in Fig. 17. The most probable pion momentum is $\approx 0.5 \mathrm{GeV} / \mathrm{c}$ but with tails out to 5 or $6 \mathrm{GeV} / \mathrm{c}$. This momentum range is well measured by long base line RICH (see Fig. 7).

The $\theta_{\mathrm{p}}$ distribution for pions is shown in Fig. 18. Most of these pions lie within our acceptance of (750-900 mrad) but a substantial tail extends out to 1.57 radians. We have not yet characterized the source of these large angle pions.

The proton momentum distribution is shown in Fig. 19. It extends out to 8 to 9 $\mathrm{GeV} / \mathrm{c}$ where our momentum resolution is stupendous i.e $\sigma_{\mathrm{p}} / \mathrm{p} \leq 1 \%$ for $\mathrm{p} \leq 10$ $\mathrm{GeV} / \mathrm{c}$. Of course we lose those below threshold however, $40 \%$ of the quasi-elastic protons are still above threshold.

The $\theta_{p}$ distribution of protons is shown in Fig. 20. Almost all protons lie within our acceptance of (750-900 $\mathrm{mrad})$.

\section{Summary and future developments}

We have shown how a RICH counter can measure momentum and have applied this method to investigate long base line neutrino oscillation experiments. This method allows large mass targets but still with measurement of momentum, direction, velocity, mass and absolute charge for charged hadrons and muons. In addition, electrons and gammas can be measured by calorirnetry with excellent energy and direction resolution. Another advantage of this method is that it uses only proven hardware i.e phototubes, mirrors and clean water

What would such a detector cost. The major elements of this detector are the PMs. Here costs are rather firm i.e $10^{3} \mathrm{SF}$ each (the SNO price) thus $\approx 15 \mathrm{MSF}$ total for the PMs. Note that PMs are reusable and even though they require an initial investment, they still have value at the end of the experiment. The cost for an above ground swimming pool of Olympic volume is estimated as 2-3 MSF Readout electronics is about $100 \mathrm{SF}$ per channel thus 1.4 MSF. Mirrors are $10^{3} \mathrm{SF} / \mathrm{m}^{2}$ and we need 900 thus 1 MSF. Water purifying and circulating system is perhaps 1 MSF. The sum comes to 21 MSF to which we add a $20 \%$ contingency for a total of $25 \mathrm{MSF}$.

We do not estimate the cost of the RICH gas counter as we are not sure it is needed and we do not yet have firm costs for HPDs. In any case, we would not install this detector in an initial stage. 


\section{Referances}

[1] J. Seguinot et al., this issue Nucl. Instr. and Meth. A??? (1996) ???.

[2] T. Ypsilantis and J. Seguinot, Nucl. Instr. and Meth. A368 (1995) 229.

[3] A. Zichichi, "Perspectives of Underground Physics: The Gran Sasso Project", CERN$\mathrm{EP} / 88-28$.

[4] Letter of Intent for Long Base Line RICH submitted 1/1/96 to Laboratorio Nazionale del Gran Sasso: "A 27 kton Liquid Water Target and RICH Photon Counter to Detect Oscillations in the CERN-Gran Sasso Neutrino Beam" Spokesman: T. Ypsilantis.

[5] T. Ypsilantis and J. Séguinot, Nucl. Instr. and Meth. A343 (1994) 30.

[6] D. Hatzifotiadou et al., CERN-LAA/PI-94-17 and College de France LPC/94-29.

[7] Y. Goldschmidt-Clermont et al., Proc. Phys. Soc. 61 (1948) 138.

[8] U. Camerini et al., Phil. Mag. 41 (1950) 413.

[9] R. Barlow, Statistics, John Wiley and Sons, section 5.2.3.

[10] R. De Salvo CLNS 87-92, Cornell University, Ithaca, NY. See also R. De Salvo et. al., Nucl. Instr. and Meth. A315 (1992) 375, Nucl. Instr. and Meth. A365 (1995) 76.

[11] I. Thormahlen, J. Straub and U. Grigull, J. Phys. Chem. Ref. Data 14 (1985) 933.

[12] A, Ball et al., CERN-ECP/95-13. To be published in Nucl. Instr. and Meth.

[13] E. Weisse, CERN, Private communication 17/11/95.

[14] Particle Data Group, Phys. Rev. D50 (1994) 1173. See page 1269. 


\section{Figure Captions}

Fig. 1. The layout of the $27 \mathrm{kton}$ water target and radiator between $\mathrm{z}=0$ to $\mathrm{z}=36$ with $x= \pm 15 \mathrm{~m}, \mathrm{y}= \pm 15 \mathrm{~m}$. A mirror of curvature $r_{m}=36 \mathrm{~m}$ is at position $\mathrm{z}=36 \mathrm{~m}$. Behind the water radiator is a $20 \mathrm{~m}$ thick gas $\mathrm{RICH}$ to measure the momentum and velocity of high energy muons.

Fig. 2. The geometry of a mirror focused ring image is defined by the mirror center of curvature $C$, the particle direction $p$ and the $Z$ axis along the neutrino beam. The PQR axes are centered on $C$ and defined so that $P$ is parallel to $\vec{p}$ and $Q$ intercepts the track, thus $\vec{p}$ is in the PQ plane and has PQR coordinates $\left(z_{2}, x_{e}, 0\right)$.

Fig. 3. Ring images of a $40 \mathrm{GeV}$ neutrino interaction $v_{\mu \rho} \rightarrow \mu^{-} \rho^{+} \eta^{\prime} \Delta^{+} \pi^{0}, \rho^{+} \rightarrow \pi^{+} \pi^{0}$, $\Delta^{+} \rightarrow \pi^{+} \pi^{0}, \eta^{\prime} \rightarrow \gamma \rho^{0} \rightarrow \gamma \pi^{+} \pi^{-}$in $25 \mathrm{~m}$ of an Argon gas radiator with $\gamma_{t}=10$. The three above thresold tracks are labeled 1, 2, 3 along with their identity and energy.

Fig. 4 (a, b) Distributions of generated Cherenkov angles for tracks $(2,3)$ of Fig.3, (c, d) the corresponding reconstructed Cherenkov angles.

Fig. 5. The Cherenkov angle width vs impact parameter xe for a $1 \mathrm{Gev} / \mathrm{c}$ pion track 85 $\mathrm{cm}$ long in water. The ontributions shown are $\sigma_{\theta}(\mathrm{ms})$ from multiple scattering, $\sigma_{\theta}(E)$ chromatic, $\sigma_{\theta}(x y z)$ from pixel size, $\sigma_{\theta}(s)$ from energy loss, $\sigma_{\theta}\left(z_{\varepsilon}\right)$ from tracklength and $\sigma_{\theta}\left(x_{e}\right)$ from impact parameter.

Fig. 6. The momentum resolution $\sigma_{p} / p$ and photoelectron number $n_{p e}(\equiv N)$ for a 1 $\mathrm{Gev} / \mathrm{c}$ pion track of $85 \mathrm{~cm}$ in water vs $x_{t}$. The loss of momentum resolution is due completely accounted for by the loss of $n_{p e}$, in accord with Fig. 5

Fig. 7. The momentum resolution $\rho p / p$ vs $p$ for $(\mu, \pi, K, P)$ in water and geometry of Fig. 1. The solid curves are from multiple scattering (Eq. 15) whereas the dot-dash curves are from the $\beta$ measurement (Eq. 7) assuming mass is known.

Fig. 8. The mass resolution $\sigma_{m}$ vs $p$ for $(\mu, \pi, K, P)$ in water and geometry of Fig. 1 . The solid curves (Eq. 16) are from combined measurements of multiple scattering and $\beta$.

Fig. 9. The momentum resolution $\sigma_{\mathrm{p}} / \mathrm{p}$ from Eq. 7 and mass resolution $\sigma_{\mathrm{m}}$ from Eq. 16 vs $\mathrm{p}$ for muons in $\mathrm{C}_{2} \mathrm{~F}_{6}$ gas $\mathrm{RICH}$ and geometry of Fig. 1. 
Fig. 10. A simulated image of a $1 \mathrm{GeV} / \mathrm{c}$ pion in water and geometry of Fig. 1. The pion is created on axis at $z_{v}=20.75 \mathrm{~m}$ in direction $\theta_{p}=0$ and $x_{e}=0$ with only emission point $\sigma_{\theta}\left(z_{e}\right)$ errors, i.e photons are emitted uniformly along the pion path $(1 \lambda=85 \mathrm{~cm})$. The $40 \mathrm{~cm}$ wide bins roughly approximates the multiple scattering contribution.

Fig. 11. Same as for Fig. 10 but the pion is created on axis at $z_{v}=20.75 \mathrm{~m}$ with $\theta_{p}=300$ mrad and $x_{e}=6.13 \mathrm{~m}$ (Eq. 21).

Fig. 12. A $5 \mathrm{GeV}$ electron shower ring image simulated in the geometry of Fig. 1. The electron starts at $z=25.2 \mathrm{~m}$ in direction $\theta_{\mathrm{p}}=0$ and $\mathrm{x}_{\mathrm{e}}=0$. Ten images are superposed but each with $\mathrm{N} / 10$ photons so the $\mathrm{N}$ value is correct but the distributions are smoothed.

Fig. 13. The radius distribution of the $5 \mathrm{GeV}$ electron shower simulation of Fig. 12. Note that the width is still sharp i.e FWHM $=200 \mathrm{~cm}$ or $\sigma_{\theta} \approx 48 \mathrm{mrad}$ but much larger and with more tails than a hadron image.

Fig. 14. The radius distribution of a simulated single $5 \mathrm{GeV}$ shower (with $\mathrm{N}$ reduced by 10). Note that the width and tails agree with the smoothed distribution of Fig. 13.

Fig. 15. A view of the showering electrons from the $5 \mathrm{GeV}$ electron shower simulation of Fig. 12.

Fig. 16. The depth distribution of the showering electrons (from the $5 \mathrm{GeV}$ electron shower of Fig. 12) showing the $Z$ origin of the imaged photons. The shower is largely spent in $5 \mathrm{~m}\left(13.9 \mathrm{X}_{0}\right)$ and completely spent in $6 \mathrm{~m}\left(16.7 \mathrm{X}_{0}\right)$.

Fig. 17. The momentum distribution $p$ of pions produced by $12 \mathrm{GeV} / \mathrm{c} v_{\mu}$ 's interacting via charged and neutral currents. Solid curves are for all pions and the dotted curves are for those above water threshold.

Fig. 18. The angular distribution $\theta_{\mathrm{p}}$ of pions produced by $12 \mathrm{GeV} / \mathrm{c} v_{\mu}$ 's interacting via charged and neutral currents. Solid curves are for all pions and the dotted curves are for those above water threshold.

Fig. 19. The momentum distribution $p$ of protons produced by $12 \mathrm{GeV} / \mathrm{c} v_{\mu}$ 's interacting via charged and neutral currents. Solid curves are for all protons and the dotted curves are for those above water threshold. 
Fig. 20. The angular distribution $\theta_{\mathrm{p}}$ of protons produced by $12 \mathrm{GeV} / \mathrm{c} v_{\mu}$ 's interacting via charged and neutral currents. Solid curves are for all protons and the dotted curves are for those above water threshold. 



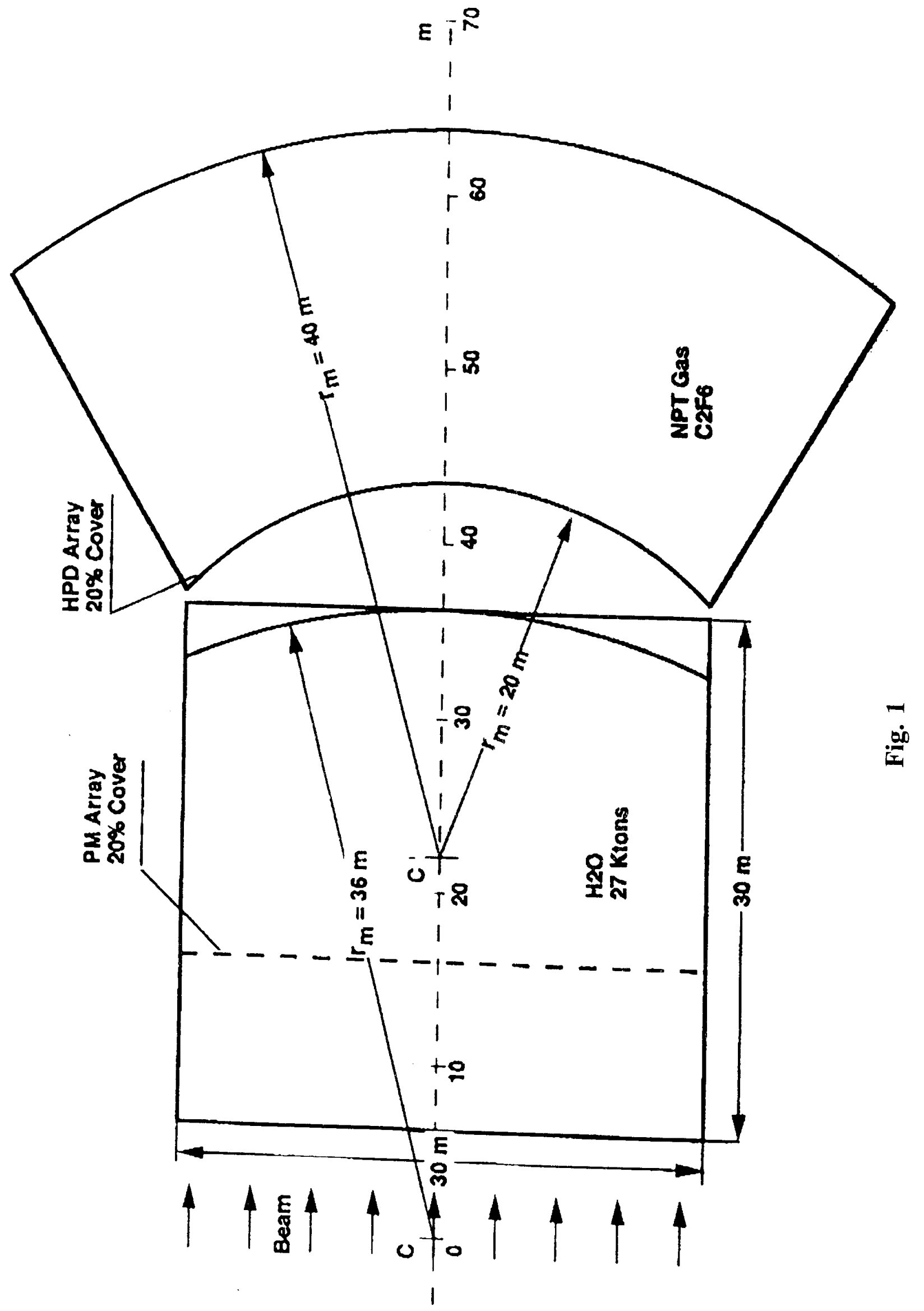




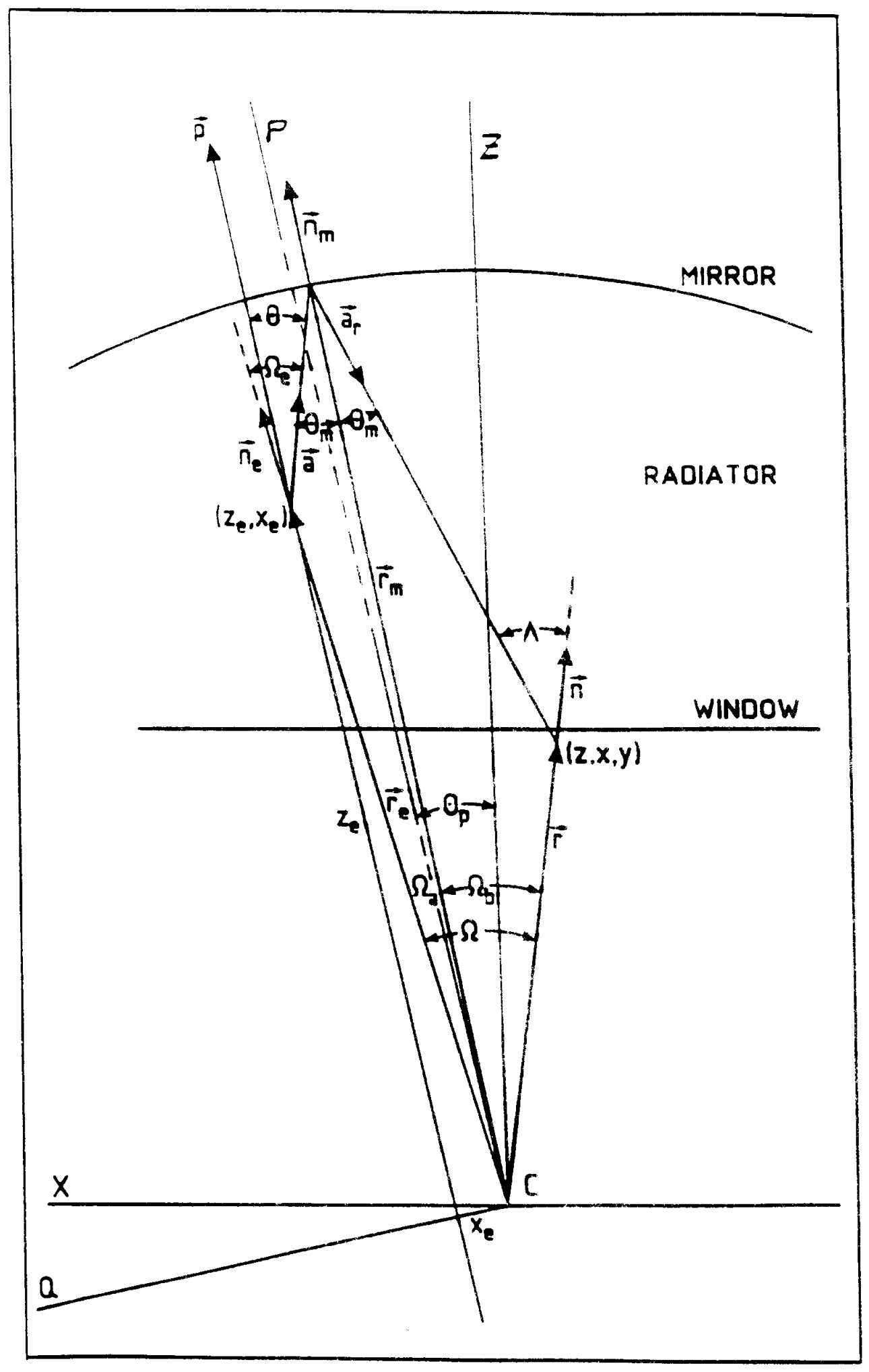

Fig. 2 


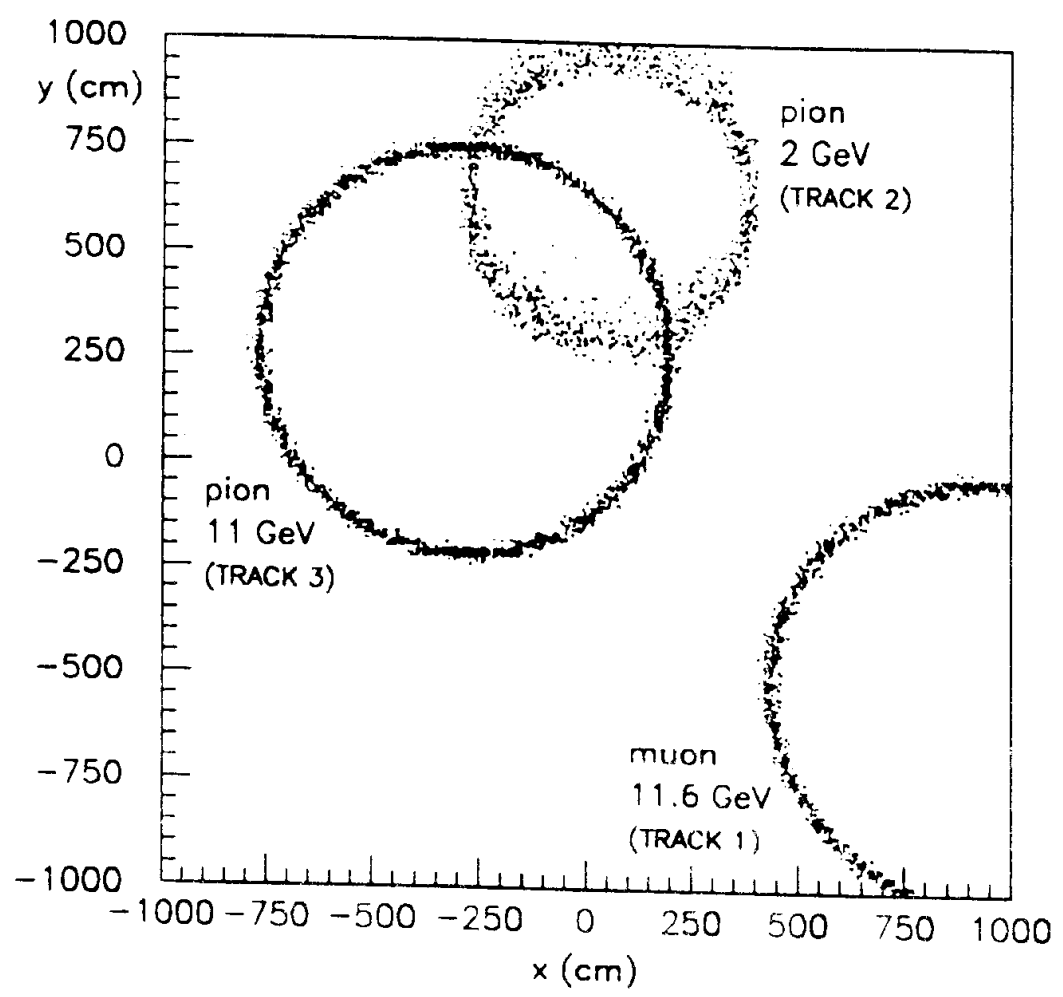

Fig. 3 

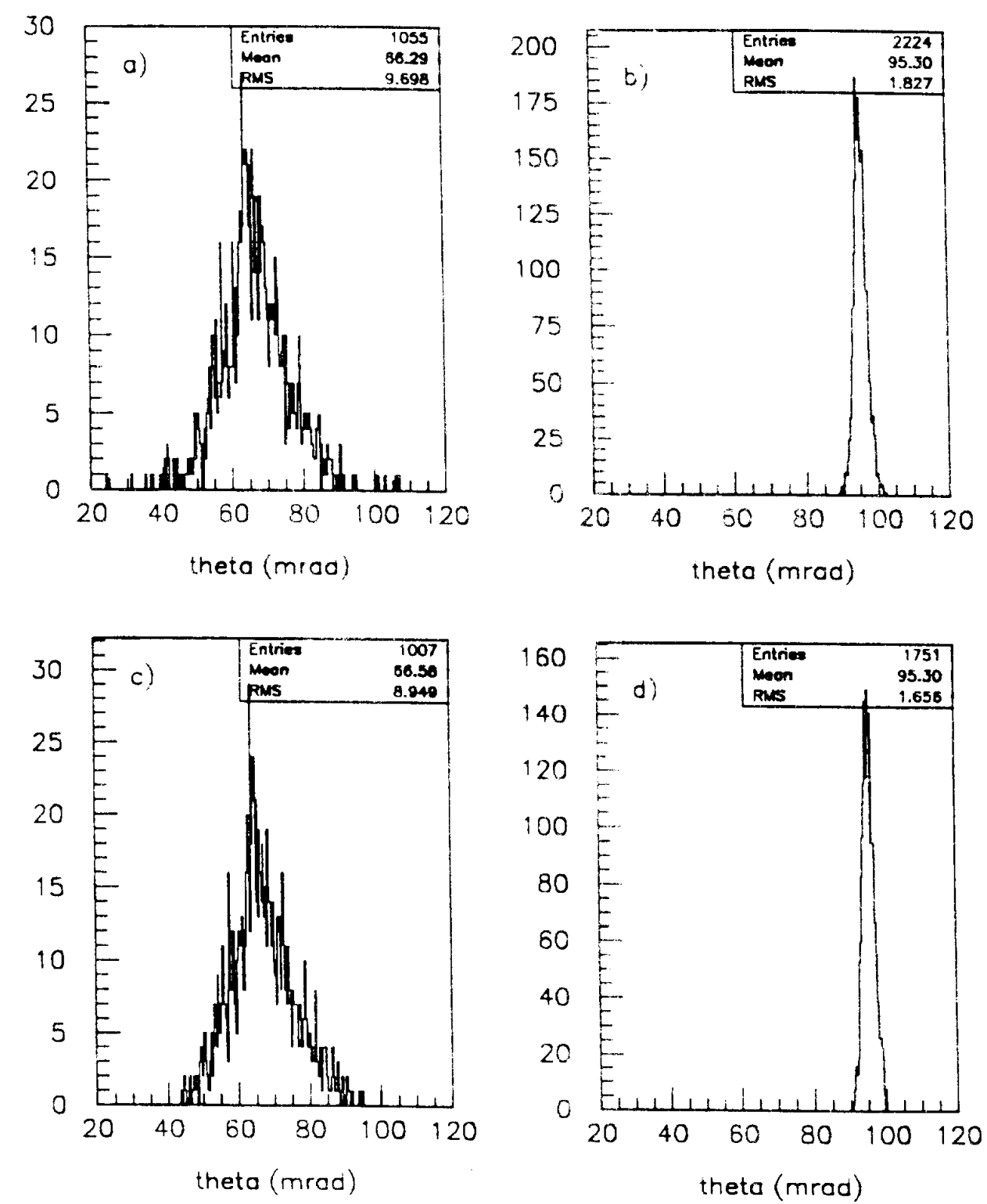

Fig. 4 


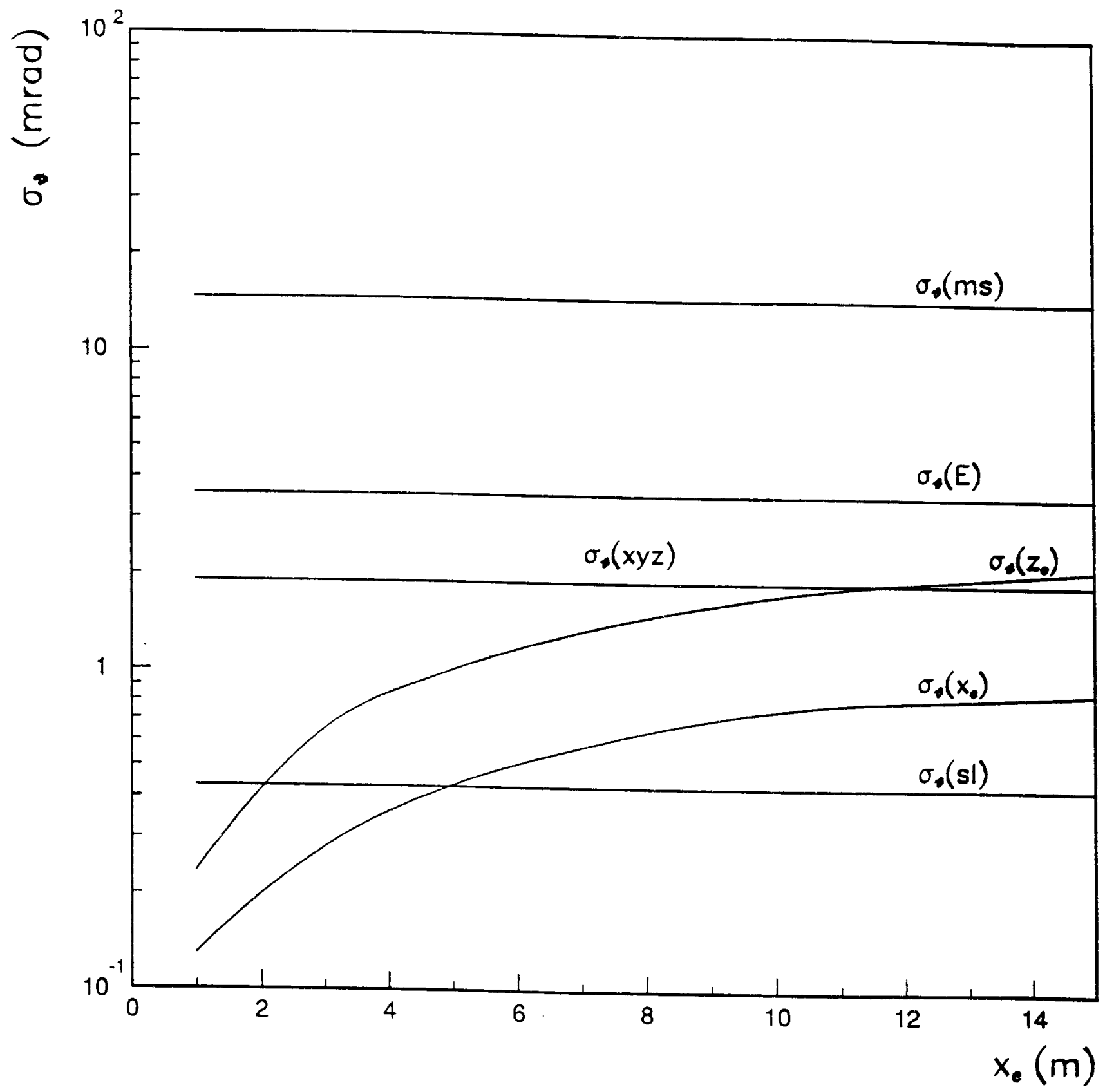

Fig. 5 


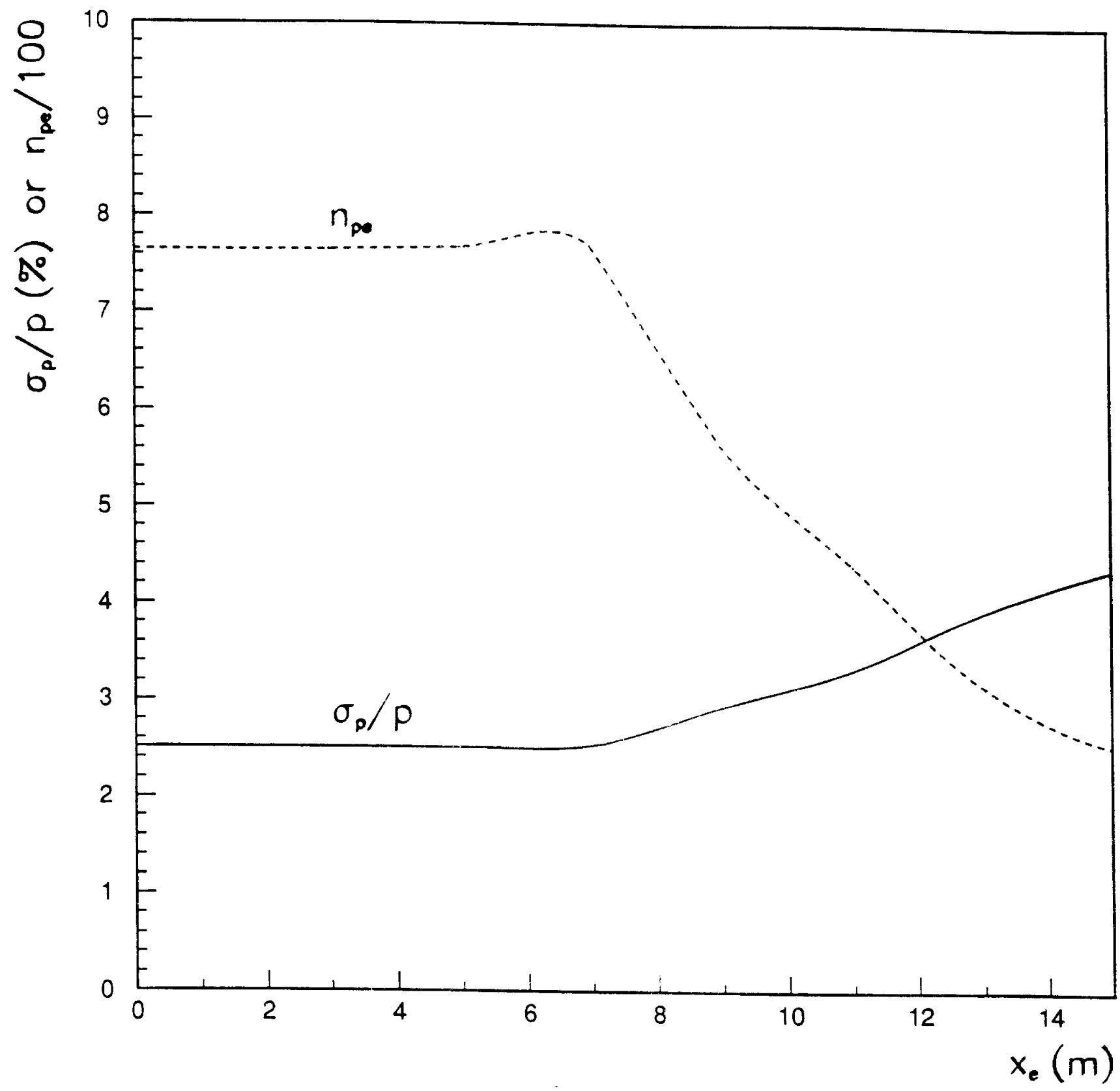

Fig. 6 


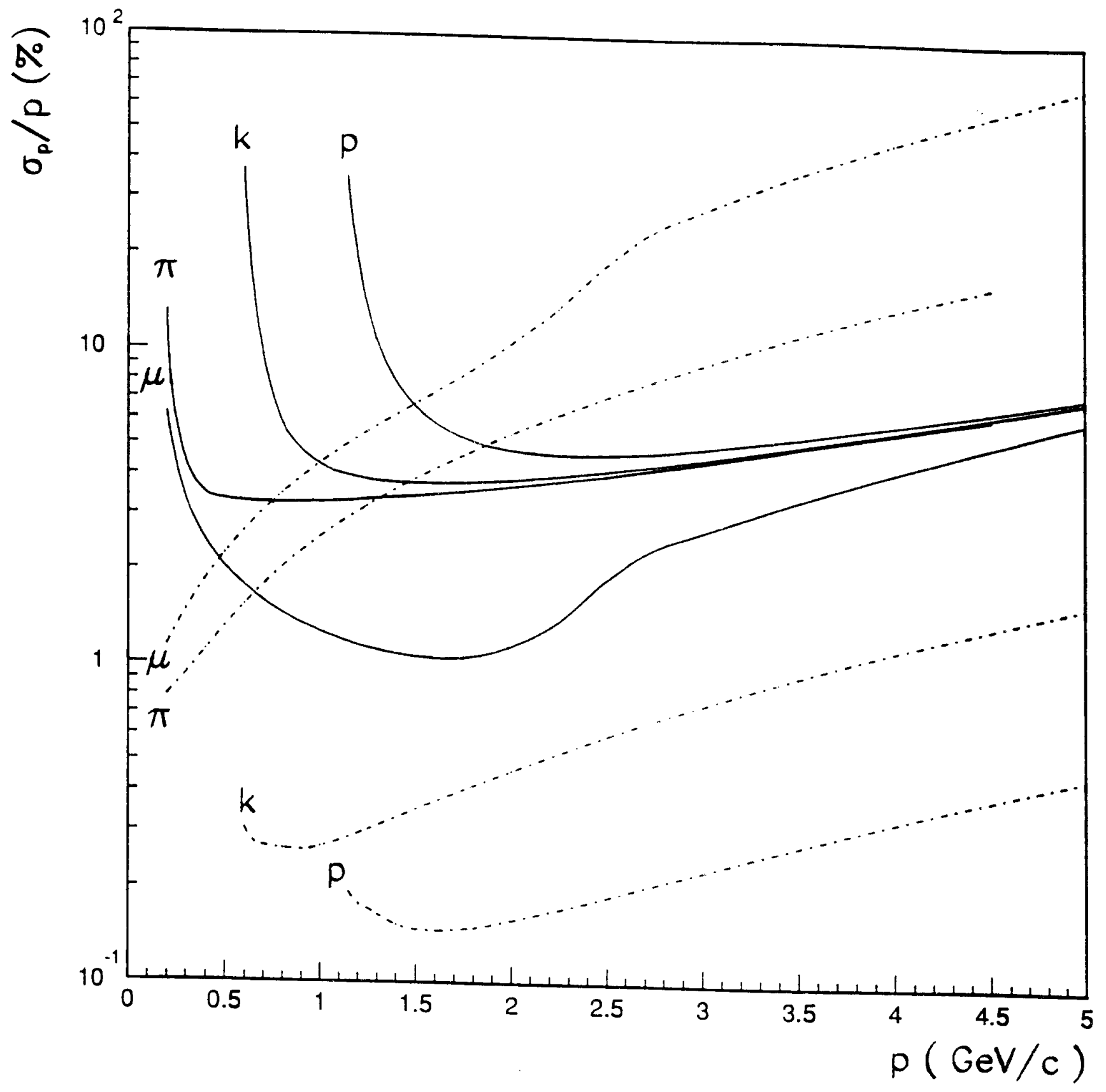

Fig. 7 


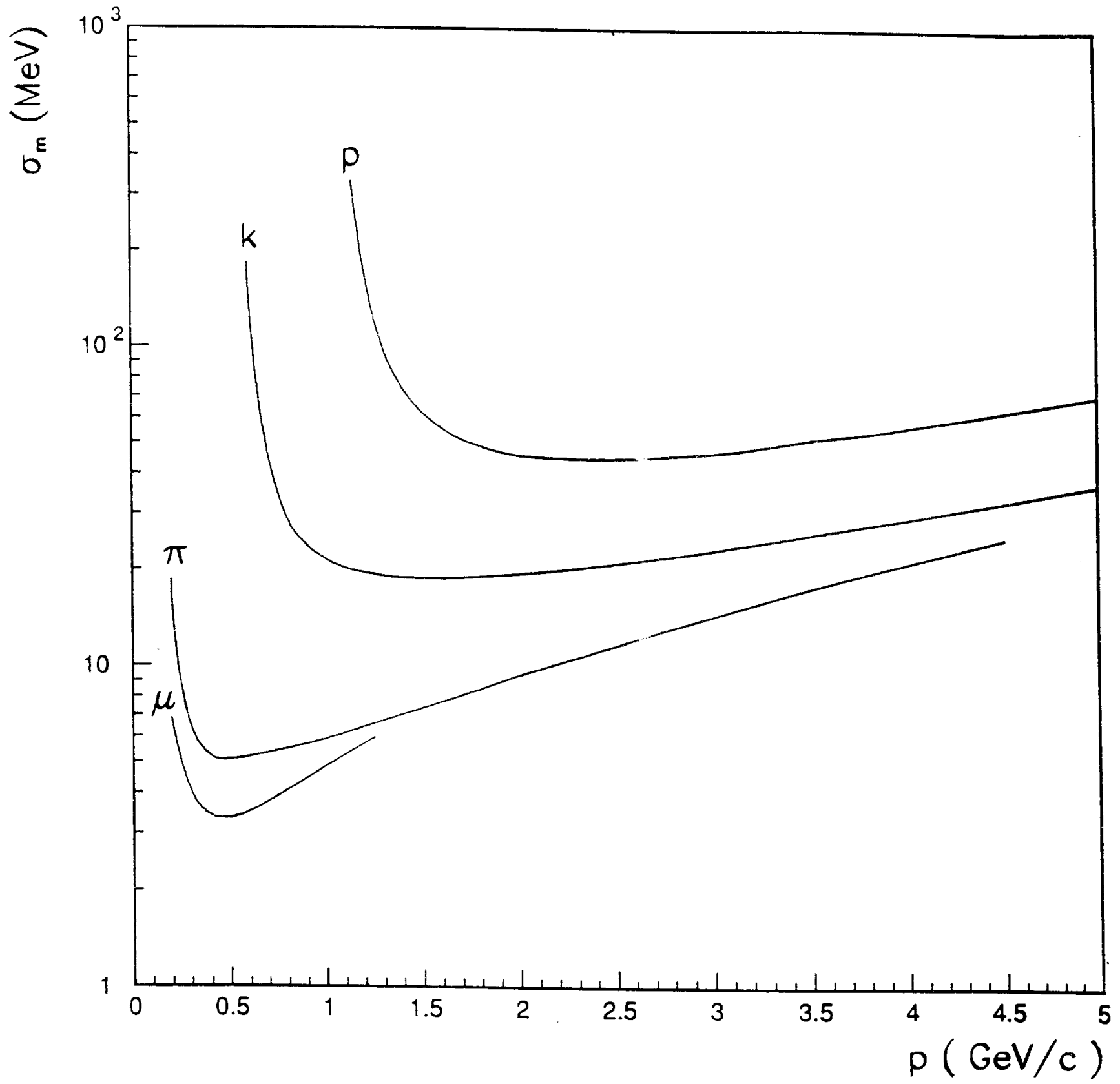

Fig. 8 


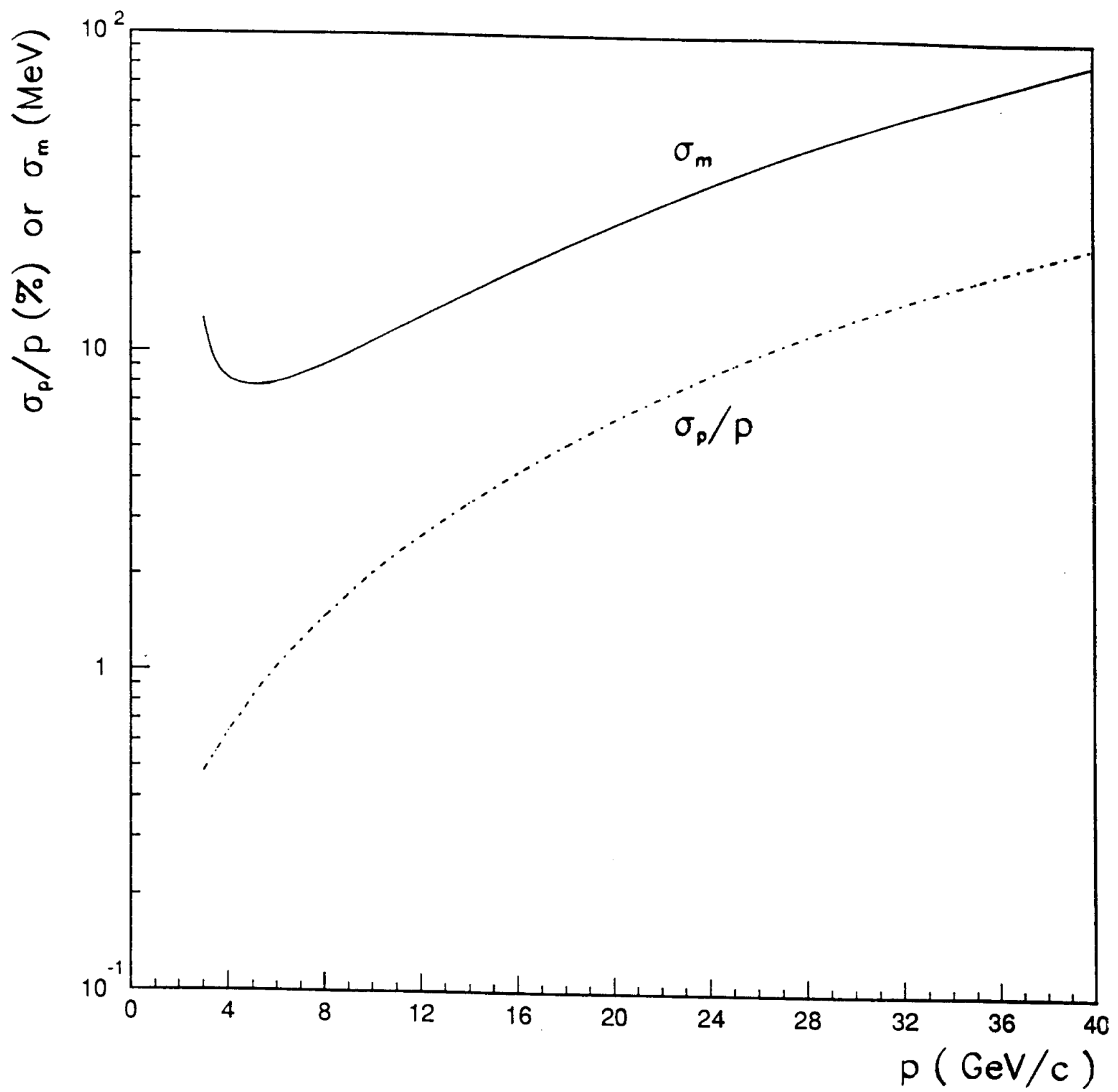

Fig. 9 


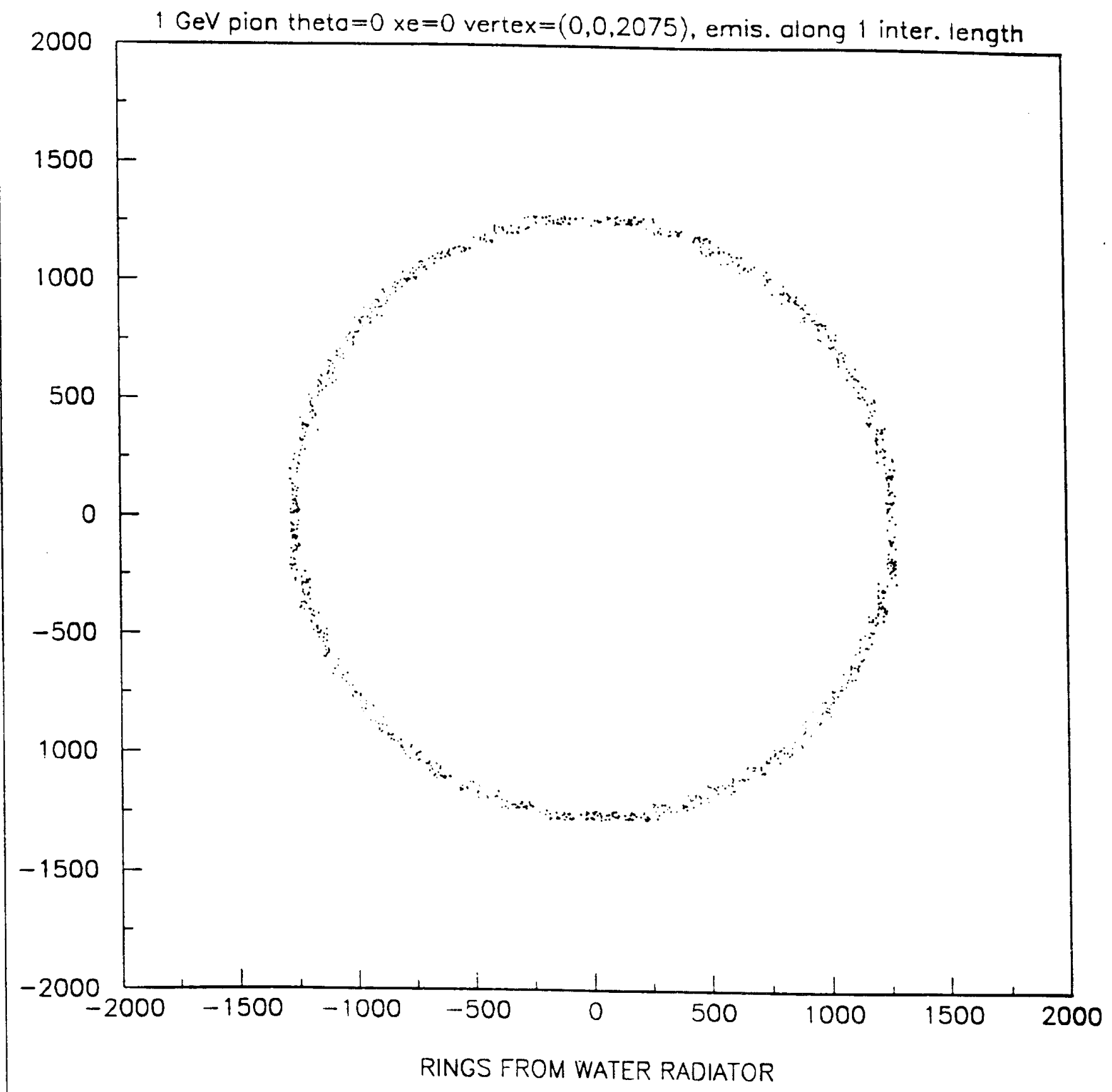

Fig. 10 


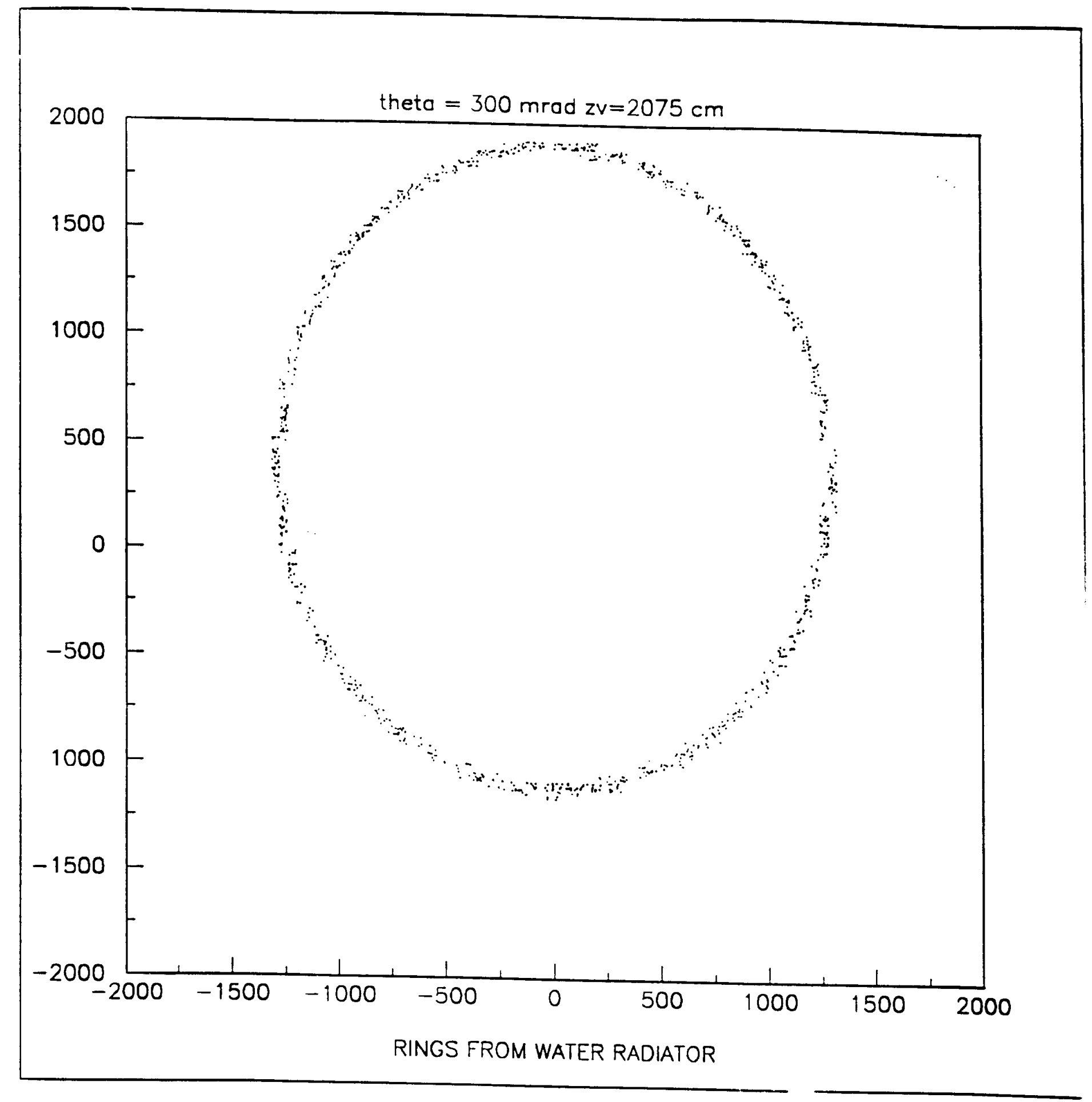

Fig. 11 
10 events Normal incidence

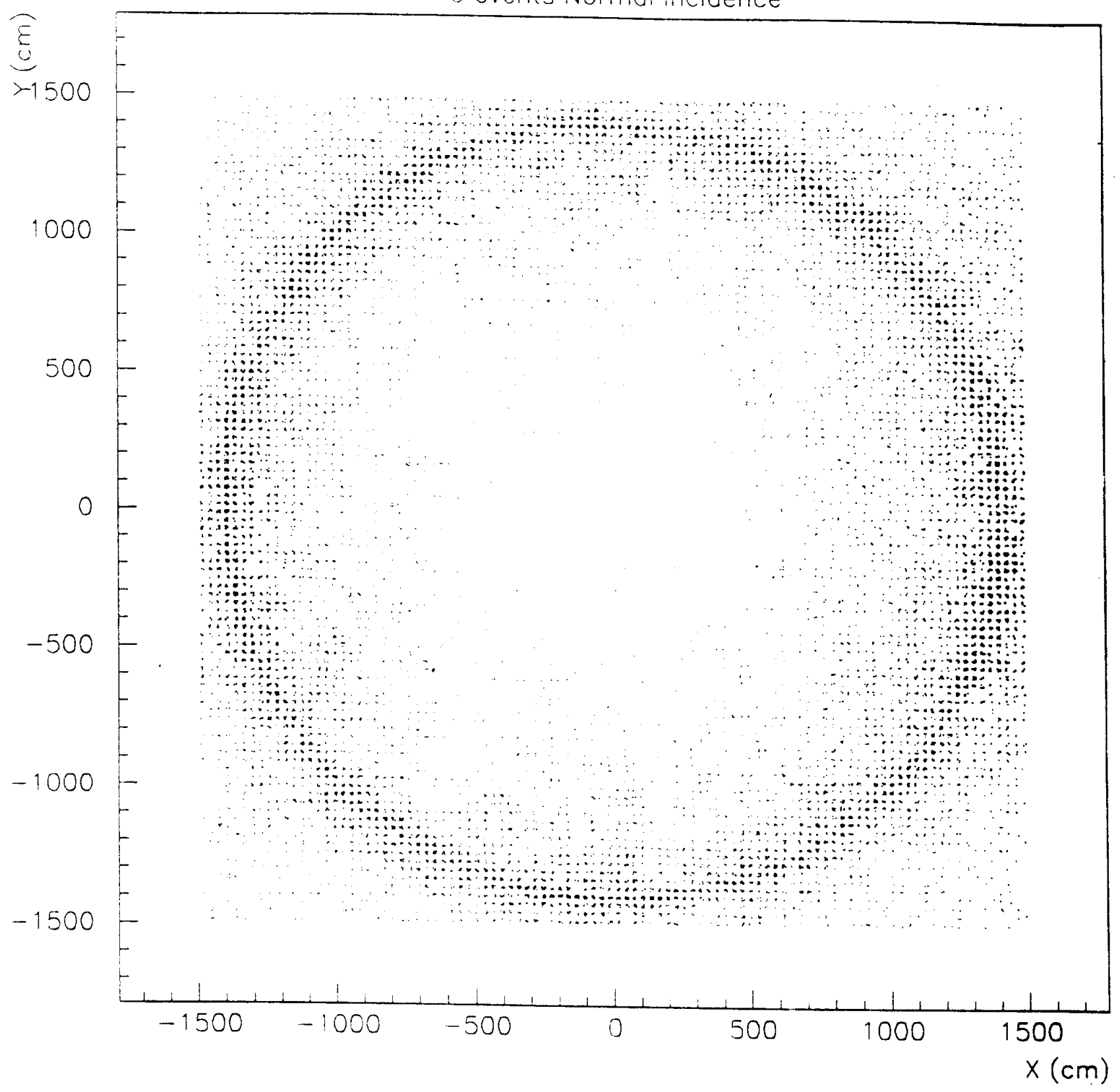

Fig. 12 


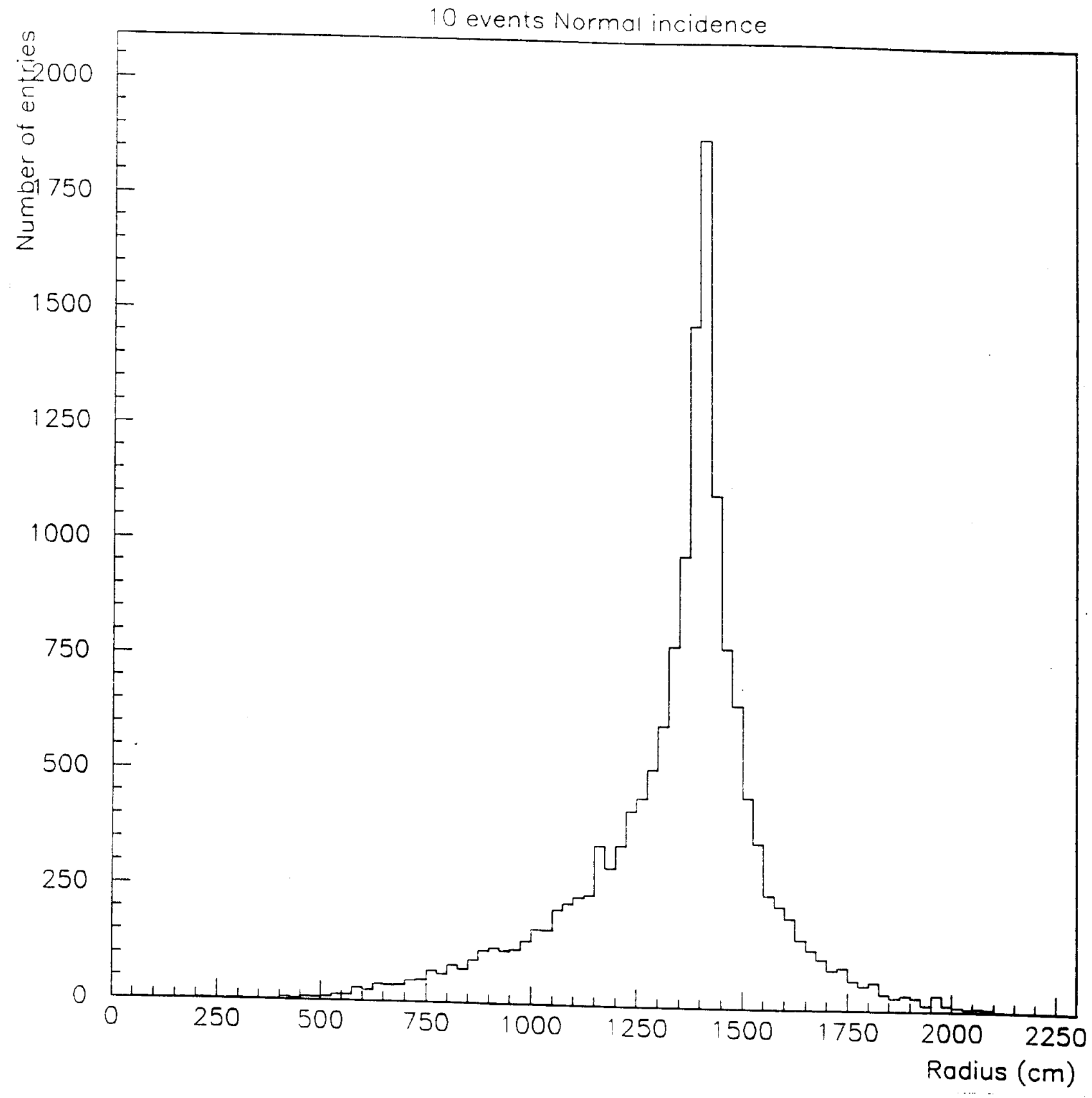

Fig. 13 


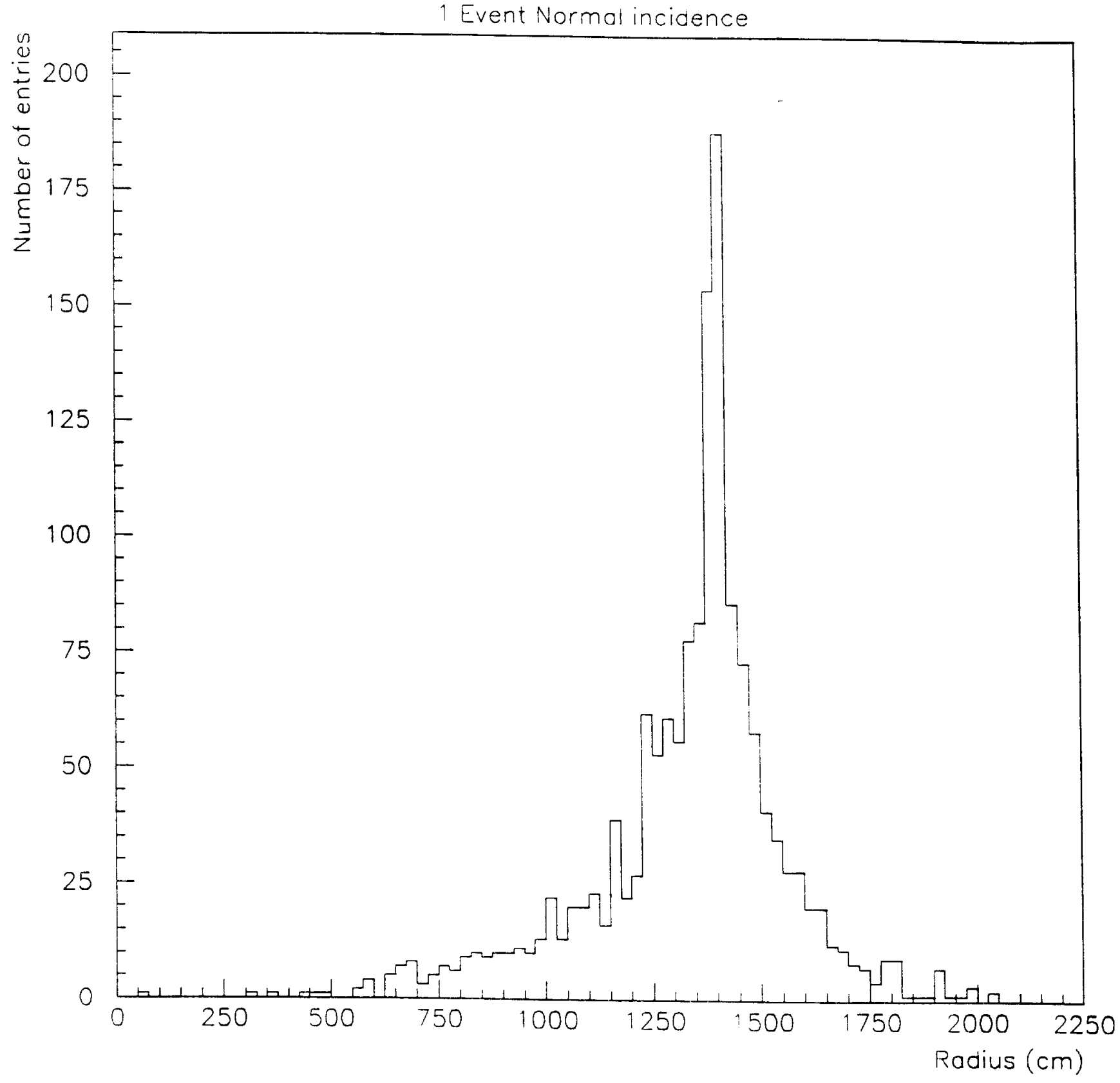

Fig. 14 
10 Events Normal incidence

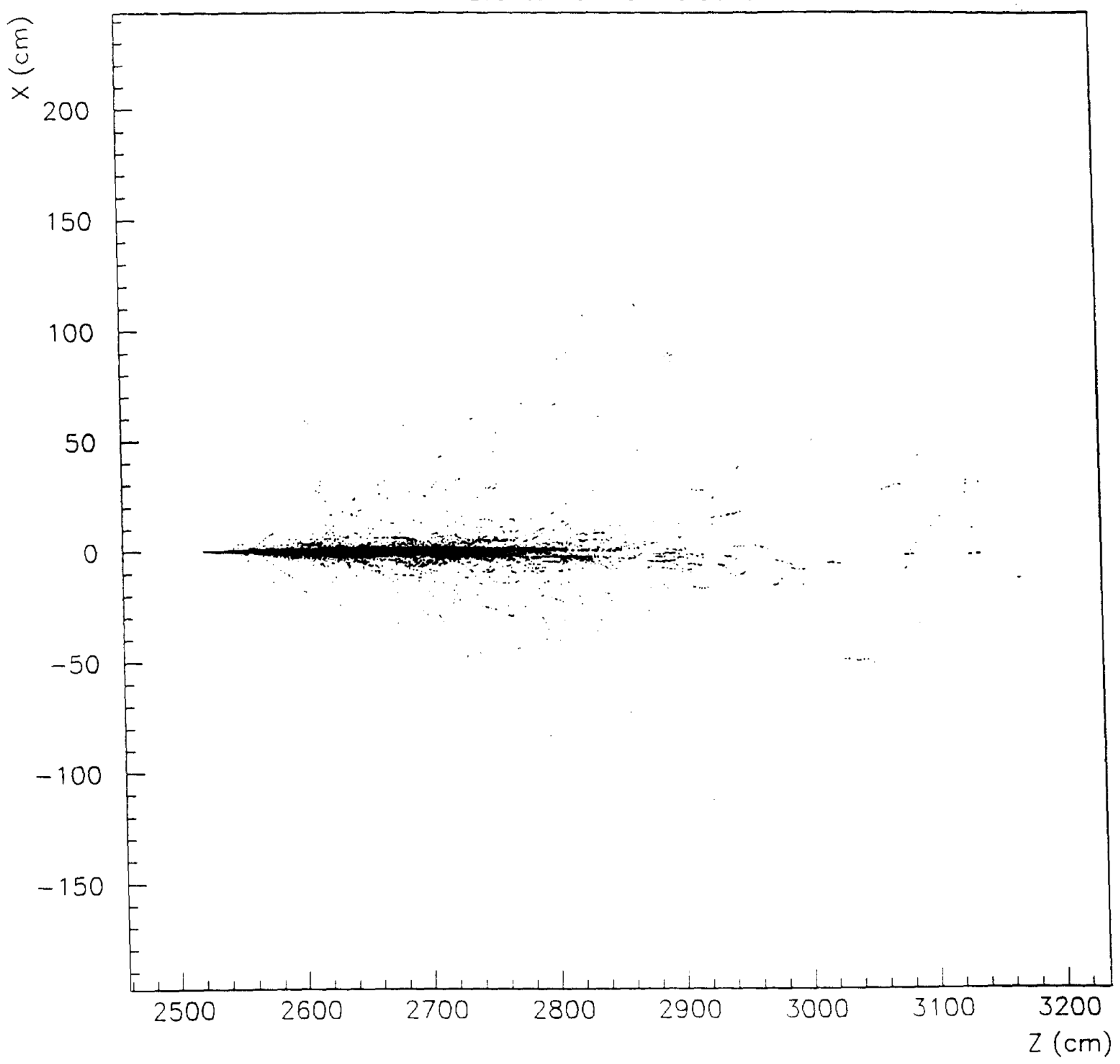

Fig. 15 


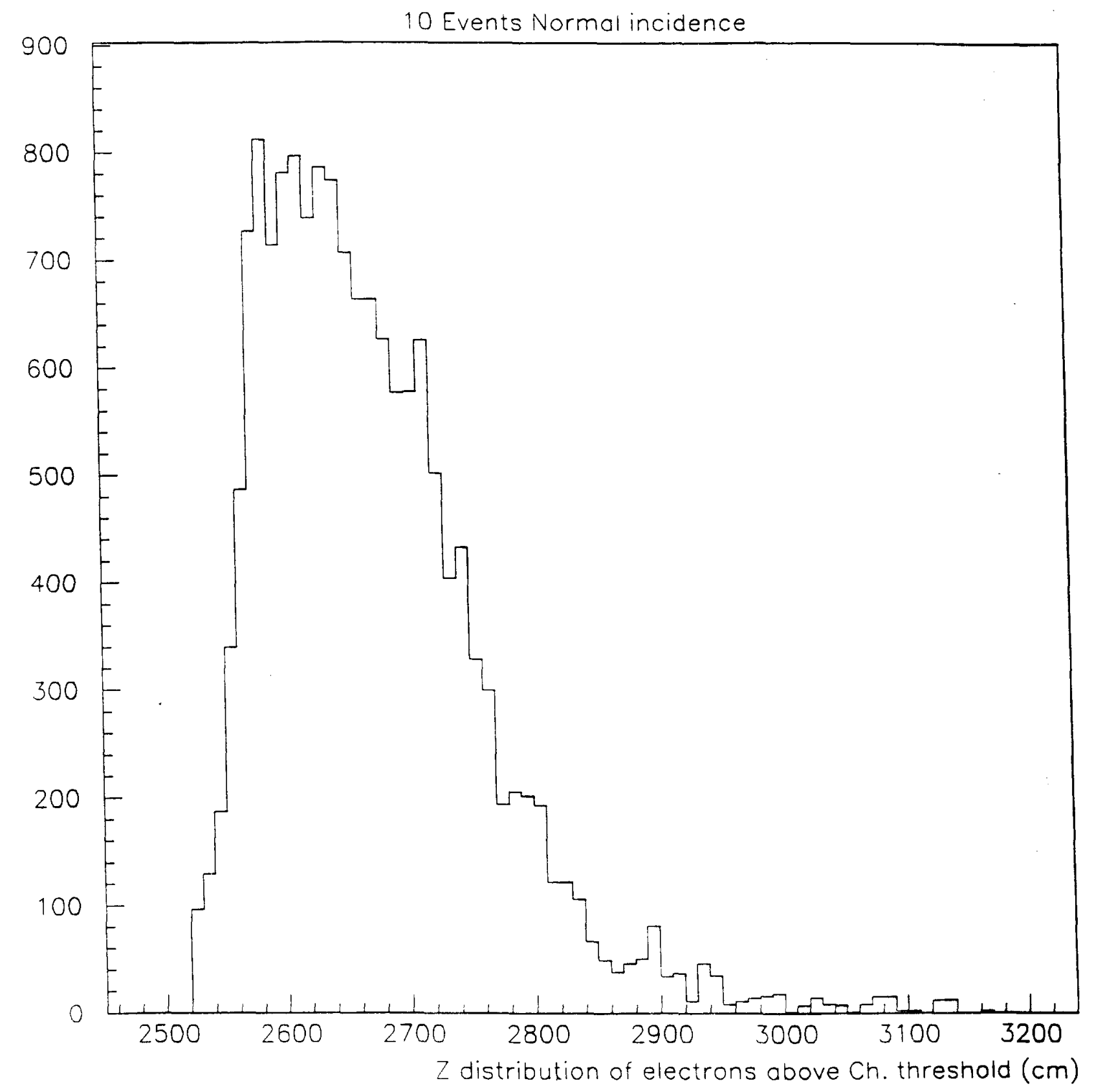

Fig. 16 


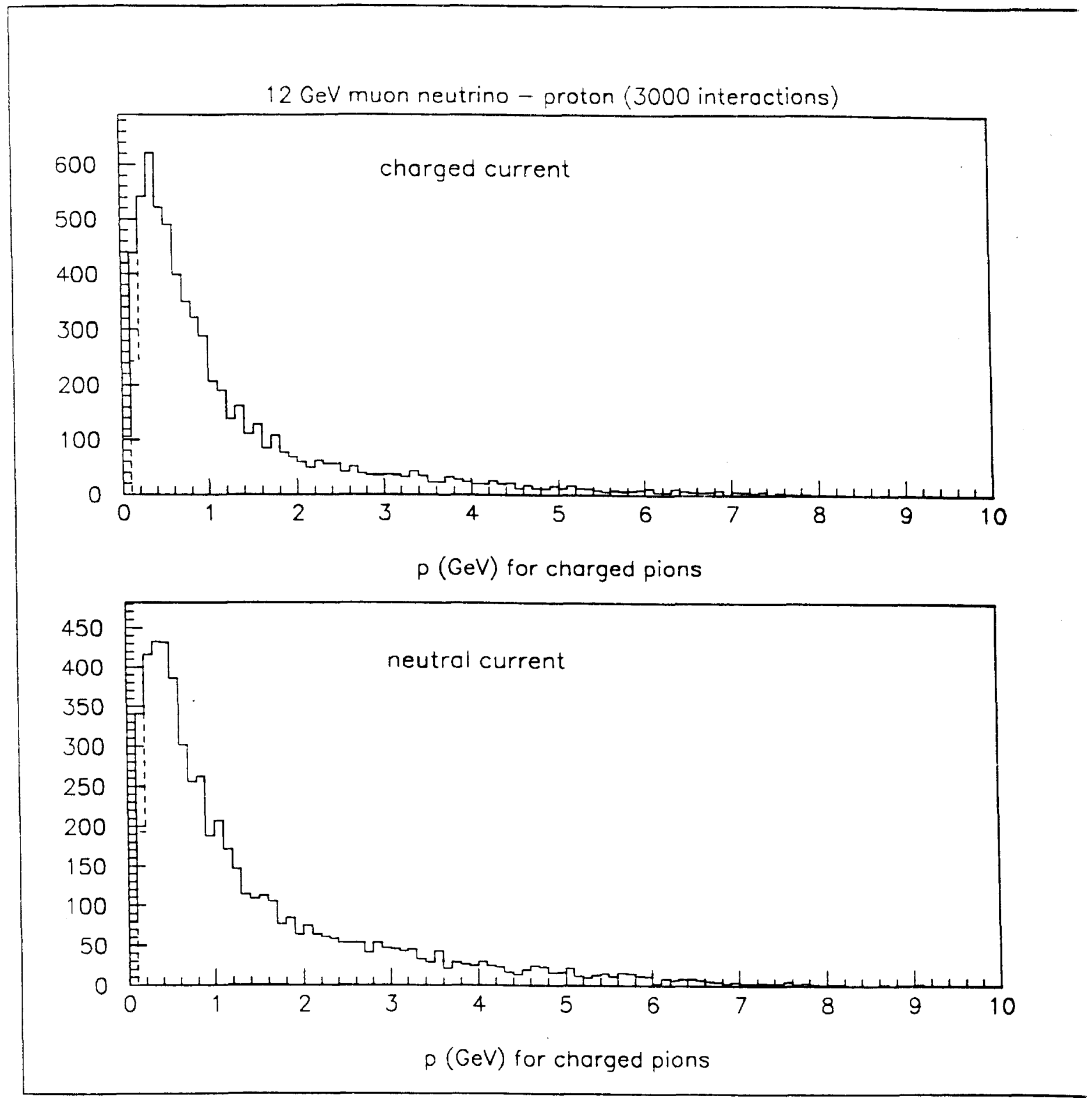

Fig. 17 


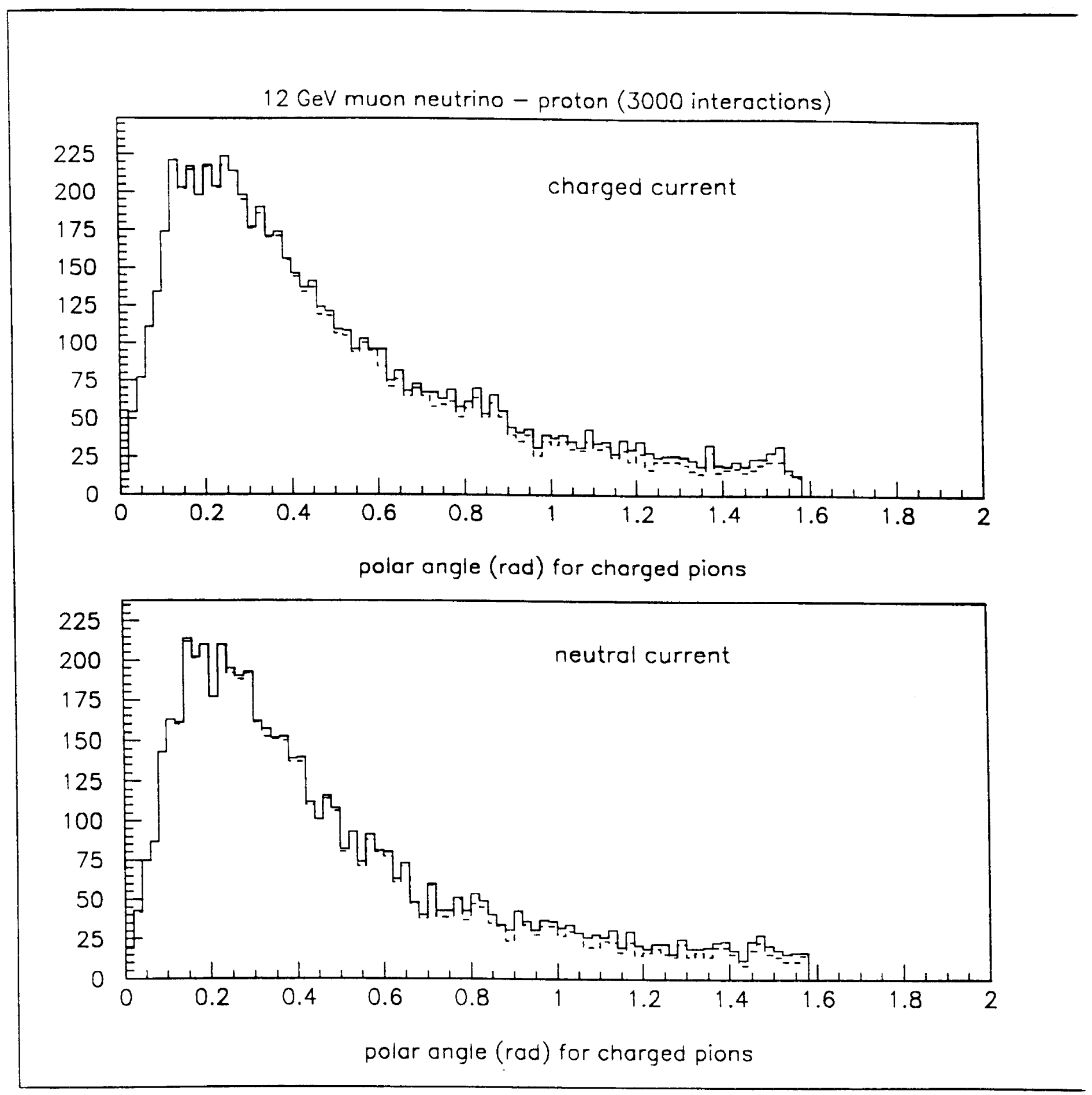

Fig. 18 

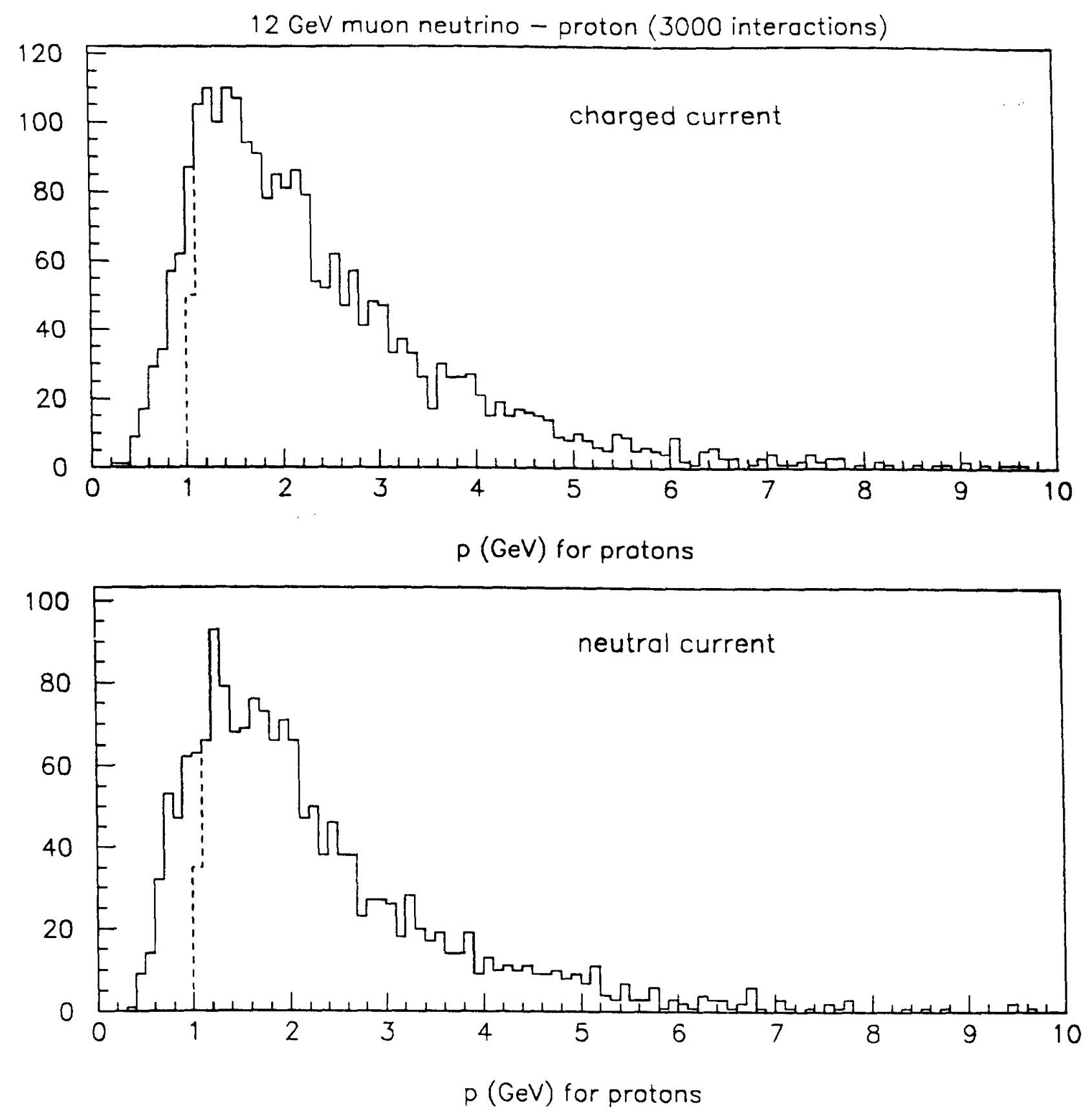

Fig. 19 

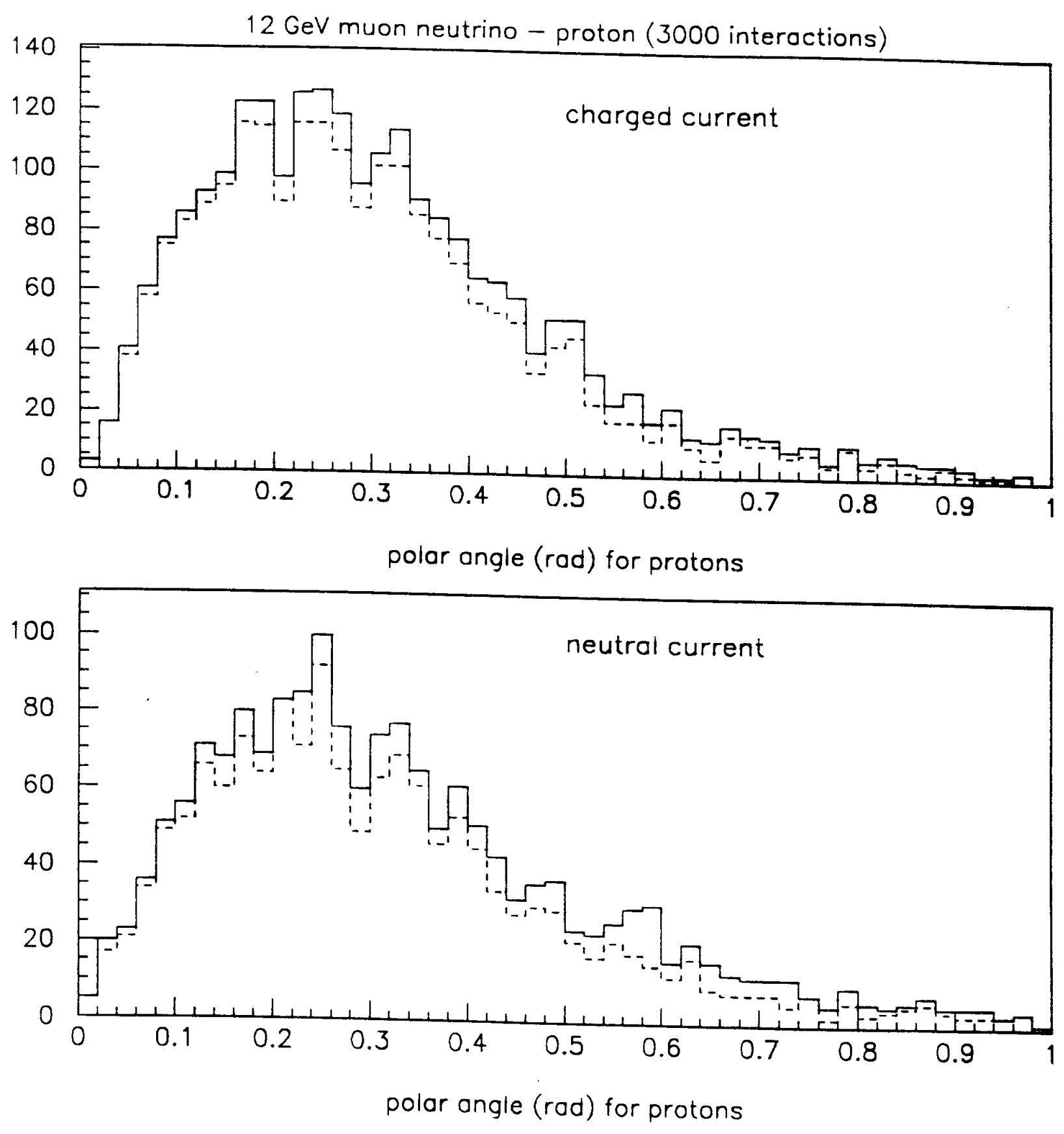

Fig. 20 


\title{
Résumé
}

Vuss proposons d'utiliser au (iran Sasso une cible de 27 ktonnes d'ean exposée à un faisceau de noutrinos en provenance du CERN pour réaliser une expérienre doscillations à grande distance. Les secomlaires chargées provenant des interartions des neutrinos produisent des photons C'erenkov dans l'eau qui sont visualisés comme des anneaux par un miroir sphérique.

Les photons sont détertés par 11400 photomultiplicateurs de $127 \mathrm{~mm}$ de diamètre, sensibles à un photon. In signal de cöncidence de $\approx 300 \mathrm{PM}$ s en temps aver l'éjection du faisceau par le SPS déflenche la lecture des PMs pendant 100 us aver une précision de $1 \mathrm{~ns}$. Ceci définit une granularité effertive du détecteur de 1.44 Mpixels.

L impulsion, la direction et la masse des hadrons et dess muons sont déterminées respectivement par la largeur, le centre et le rayon des anneaux. L'impulsion est mesurée si la diffusion multiple est linfet dominant sur la largeur de l'anneau, ce qui est le cas pour la plupart des particules intéressantes. Dans l'ran, les seuils des muons, des pions, des kaons et des protons sont respectivement $0.12,0.16$, 0.55 et $1.0 .5 \mathrm{GeV} / \mathrm{c}$.

On prévoit des résolutions de 1 à $10 \%$ sur l'mpulsion, do 5 à $50 \mathrm{MeV}$ sur la masse el inférieures à 1 mral sur la direction. Les électrons et les photons penvent ẹtre mesurés avec une résolution en cuergie $d e \sigma_{\mathrm{E}} / \mathrm{E} \approx 7 \% / \sqrt{\mathrm{E}}(\mathrm{GeV})$ et aver une résolution angulaire d'environ $1 \mathrm{mrad}$.

Le faisceau SPS est pulsé de telle sorte que le rejet du fond des rayons cosmiques est excellent et par conséquent, il est inutile d'enterrer lo détecteur.

\begin{abstract}
A 27 kton water volume is considered as a target for a long base line neut rino beam from CERN to (iran Sasso. (tharged secondaries from the neutrino interactions produce Cerenkov photons in water which are imaged as rings by a spherical mirror.

The photon detector elements are 14400 photomultipliers (PMs) of $127 \mathrm{~mm}$ diameter with single photon sensitivity. A coincidence signal of $\approx 300$ PMs in time with the SPS beam burst starts readout of the $\mathrm{P} M \mathrm{Ms}$ in bins of 1 ns over a period of $100 \mathrm{~ns}$. This defines the effective detector granularity to be 1.44 Mpixels, quite sufficient for the maximum expected event size of $\approx 2.10^{4}$ photon hit points.

Momentum, direction and velocity of hadrons and muons are determined from the width, center and radius of the rings, respectively. Momentum is measured if multiple scattering dominates the ring width, as is the case for most of the particles of interest. Thresholds in water for muons, pions, kanns and protons are $0.12,0.16,0.55$ and 1.05 GeV/cenespectively.

Momentum resolutions of $1-10 \%$. mass resolutions of 5-50 MeV and direction resolutions of $<1$ mrarl, are achievable. Electrons and gammas can be measured with energy resolution $\sigma_{\mathrm{E}} / \mathrm{E} \approx 7 \% / \sqrt{\mathrm{E}}$ $($ ( $\mathrm{e} V$ ) and with direction resolution $\approx 1 \mathrm{mrad}$.

The detector can be sited above ground because it is directional and the SPS beam is pulsed thiss it has excellent rejection of cosmic ray background.
\end{abstract}


